\title{
Model Predictive Control for Load Frequency Control with Wind Turbines
}

\author{
Yi Zhang, ${ }^{1,2}$ Xiangjie Liu, ${ }^{2}$ and Yujia Yan ${ }^{2}$ \\ ${ }^{1}$ Department of Electrical Engineering, North China University of Science and Technology, Tangshan 063000, China \\ ${ }^{2}$ The State Key Laboratory of Alternate Electrical Power System with Renewable Energy Sources, North China Electric Power University, \\ Beijing 102206, China \\ Correspondence should be addressed to Yi Zhang; zhangyizhouzhao@163.com
}

Received 23 August 2015; Accepted 12 October 2015

Academic Editor: Onur Toker

Copyright (C) 2015 Yi Zhang et al. This is an open access article distributed under the Creative Commons Attribution License, which permits unrestricted use, distribution, and reproduction in any medium, provided the original work is properly cited.

Reliable load frequency (LFC) control is crucial to the operation and design of modern electric power systems. Considering the LFC problem of a four-area interconnected power system with wind turbines, this paper presents a distributed model predictive control (DMPC) based on coordination scheme. The proposed algorithm solves a series of local optimization problems to minimize a performance objective for each control area. The scheme incorporates the two critical nonlinear constraints, for example, the generation rate constraint (GRC) and the valve limit, into convex optimization problems. Furthermore, the algorithm reduces the impact on the randomness and intermittence of wind turbine effectively. A performance comparison between the proposed controller with and that without the participation of the wind turbines is carried out. Good performance is obtained in the presence of power system nonlinearities due to the governors and turbines constraints and load change disturbances.

\section{Introduction}

Wind energy is considered as a promising and encoring renewable energy alternative for power generation owing to environmental and economical benefits. The world market of wind installation set a new record in the year of 2014 and reached a total size of $51 \mathrm{GW}$ [1]. Nowadays, due to the interconnection of more distributed generators, especially wind turbines that are committed to grid operation, electric power system has become more complicated than ever.

Power system LFC incorporating WTGs can be a quite challengeable issue. The output power of WTGs varies with wind speed fluctuation [2]. This wind power fluctuation imposed additional power imbalance to the power system and may cause frequency deviation from the nominal value [3]. Significant frequency deviations may lead to the disconnection of some loads and generations and even can lead to whole power system oscillations. Previous studies [4-6] provide extensive overviews of the primary and secondary frequency control strategies of power systems with wind power plants.
LFC, secondary frequency control, has been performed by integrating the area control error (ACE), which acts on the load reference settings of the governors. LFC tasks are maintaining tie-line power flow and system frequency close to nominal value for the multiarea interconnected power system [7]. As a fundamental characteristic of electric power operations, frequency of the system deviates from its nominal value due to generation-demand imbalance. Conventional generators, in which the turbine rotational speed is nearly constant, provide inertia and governor response against frequency deviations; however, the speed of a wind turbine is not synchronous with the grid and is usually controlled to track the maximum power point. It implies that the wind turbines will have less time to react to the power imbalance, probably resulting in lager frequency deviations.

Thus, it is thus necessary to establish the optimal profile of the WTGs power surge in coordination with the characteristics of conventional plants to achieve a more economical and reliable operation of power system. With the large amount of realistic constraints, for example, generation rate constraints 
(GRCs) in the conventional units, the pitch angle, and generator torque constraints in WTGs, the LFC becomes a largescale, distributed, multiconstraints optimization problem.

Recently, a few attempts studied the idea of wind turbines in the issue of LFC [8-10]. Two types of wind farm models are derived and demonstrated to portray the capability of set-point tracking under automatic generation control (AGC) [8]. This inference leads to the development of a simplified wind farm model that is specially designed for the set-point control in the power system study. However, the durability of inertia effect depends on the allowable rotor speed range. An adaptive fuzzy logic structure was used to propose a new LFC scheme in the interconnected large-scale power system in the presence of wind turbines [9]. The performance against sudden load change and wind power fluctuations in different wind power penetration rates is confirmed by simulation. A flatness-based method to control frequency and power flow for multiarea power system with wind turbine is presented in [10]. And, practical constraints such as generator ramping rates of wind turbine generator can be considered in designing the controllers. As abovementioned reference, the control schemes are designed for each area to maintain the frequency at nominal value and to keep power flows near scheduled values. However, local controller in each area does not work cooperatively towards satisfying systemwide control objectives. In addition, the control scheme [8-10] mentioned above could yield unsatisfactory performance since the effects of nonlinearities such as generation rate constraint and generation ramping rate were not considered.

Model predictive control (MPC), also called receding horizon control, was originally developed to be an effective method for processing industrial control. It transforms the control problem into a finite horizon optimal control problem that can also satisfy multivariable constraints on the input, output, and state variables. In the power industry, MPC has been successfully used in controlling power plant steamboiler generation processes [11-13]. In power system control, MPC was first developed to be an economic-oriented LFC [14], which generates the control action based on the open-loop optimization method over a finite horizon. MPC has subsequently been developed to realize the constrained optimal algorithm for LFC problem. In [15], the constraint handling ability of MPC is employed to effectively account for the generation rate constraints (GRCs) but without the analysis of closed-loop stability and robustness. Recently, MPC has been successfully used in LFC design of multiarea power system with wind turbines [16]. However, each area controller is designed independently and the communication between the local controllers is not considered. On the other hand, with the size and capacity of wind farms increasing in recent years, traditional centralized MPCs encounter many difficulties due to limitations in exchanging information with large-scale, geographically extensive control areas. In order to deal with these issues, advanced distributed control strategies have to be investigated and implemented.

Developing decentralized/distributed LFC structures can be an effective way of solving this problem. The decentralized model predictive control scheme for the LFC of multiarea interconnected power system is presented in [17]. However, the local controller does not consider generation rate constraint that is only imposed on the turbine in the simulation. In the distributed MPC (DMPC), the benefits from using a decentralized structure are partially preserved, and the plant-wide performance and stability are improved through coordination [18, 19]. In [20], feasible cooperation-based MPC method is used in distributed LFC instead of centralized MPC. It is noted that the range of load change used in the cases is very large and inappropriate for the LFC issue.

This paper studies the effect of merging the wind turbines on the system frequency of multiarea power system. The first control area includes an aggregated wind turbine model (which consists of 60 wind turbine units) beside the thermal power plant. According to the distributed LFC structure, the dynamics model of the four-area interconnected power system is established. In our scheme, the overall power system is decomposed into four areas and each area has its own local MPC controller. These areas-based MPCs exchange their measurement and predictions by communication and incorporate the information from other controllers into their local objective so as to coordinate with each other. The controllers calculate the optimal control signal while respecting constraints over the wind turbines output frequency deviation and the load change. Not only do the effects of the physical constraints conclude generating rate constraints (GRCs) and the limit of governor position in conventional power plant, but also the wind speed constraints in wind turbines are considered. Comparisons of response to step load change, computational burden, and robustness have been made between DMPC, centralized MPC, and decentralized MPC. The results confirm the superiority of the proposed DMPC technique.

The remainder of the paper is organized as follows. Modeling of wind turbines participation in LFC is presented in Section 2, and the proposed DMPC algorithm is presented in Section 3. Section 4 presents the application of the algorithm in a four-area interconnected power system. The conclusions are presented in Section 5.

\section{Distributed Model of Hybrid Power System}

Figure 1 illustrates the interconnected power system consisting of four control areas connected by tie-lines, which consists of thermal power plant, variable speed wind turbines (VSWTs), and hydro power plant. In area 1, wind turbine is taken into consideration as it can provide a new solution to the contradiction between economic development and environment pollution. Area 4 is the thermal power plant, while area 2 and area 3 are hydro power plants.

Detailed compositions of each area are shown in Figures $2-4$. In addition, area 1 includes an aggregated wind turbine model which consists of 40 VSWT units while the capacity of thermal plant is $600 \mathrm{MW}$. The variables and parameters are listed in Table 1 . In each control area, a change in local demand (load) alters the nominal frequency. The DMPC in each control area $i$ manipulates the load reference setpoint $\Delta P_{\text {ref }, i}$ to drive the frequency deviations $\Delta f_{i}$ and tie-line power flow deviations $\Delta P_{\text {tie }, i j}$ to zero. 
TABLE 1: Power system variables and parameter.

\begin{tabular}{|c|c|c|}
\hline Parameter/variable & Description & Unit \\
\hline$\omega_{r}$ & Angular velocity of rotor & $\mathrm{rad} / \mathrm{s}$ \\
\hline$\omega_{g}$ & Angular velocity of high speed shaft and generator & $\mathrm{rad} / \mathrm{s}$ \\
\hline$T_{g}$ & Generator reaction torque & $\mathrm{Nm}$ \\
\hline$T_{r}$ & Aerodynamic torque & $\mathrm{Nm}$ \\
\hline$K_{s}$ & Total stiffness on low speed shaft & $\mathrm{Nm} / \mathrm{rad}$ \\
\hline$J_{r}$ & Inertia of the rotor (low speed shaft and gearbox) & $\mathrm{Kgm}^{2}$ \\
\hline$J_{g}$ & Inertia of the rotor (high speed shaft and gearbox) & $\mathrm{Kgm}^{2}$ \\
\hline$n_{\text {gear }}$ & Exchange ratio & Null \\
\hline$\eta_{\text {gear }}$ & Efficiency of the gear box & $\%$ \\
\hline$\tau_{\beta}$ & Actuator time constant & s \\
\hline$K_{\beta}$ & Actuator gain & Hz/p.u.MW \\
\hline$v_{m}$ & Wind speed & $\mathrm{m} / \mathrm{s}$ \\
\hline$\theta_{\text {ref }}$ & Pitch demand & $\mathrm{rad}$ \\
\hline$\theta$ & Pitch angle & $\mathrm{rad}$ \\
\hline$P_{e}^{\text {ref }}$ & Power demand & p.u.MW \\
\hline$P_{e}$ & The output of wind turbine & p.u.MW \\
\hline$\Delta f_{i}(t)$ & Frequency deviation & $\mathrm{Hz}$ \\
\hline$\Delta P_{g i}(t)$ & Generator output power deviation & p.u.MW \\
\hline$\Delta X_{g i}(t)$ & Governor valve position deviation & p.u. \\
\hline$\Delta X_{g h i}(t)$ & Governor valve servomotor position deviation & p.u. \\
\hline$\Delta P_{t i e, i}(t)$ & Tie-line active power deviation & p.u.MW \\
\hline$\Delta P_{d i}(t)$ & Load disturbance & p.u.MW \\
\hline$K_{P i}$ & Power system gain & Hz/p.u.MW \\
\hline$K_{r i}$ & Reheat turbine gain & Hz/p.u.MW \\
\hline$T_{P i}$ & Power system time constant & s \\
\hline$T_{r i}$ & Reheat turbine time constant & s \\
\hline$T_{W i}$ & Water starting time & s \\
\hline$T_{i i}, T_{R i}$ & Hydro governor time constants & s \\
\hline$T_{G i}$ & Thermal governor time constant & s \\
\hline$T_{T i}$ & Turbine time constant & s \\
\hline$K_{S i j}$ & Interconnection gain between control areas & p.u.MW \\
\hline$K_{B i}$ & Frequency bias factor & p.u.MW/Hz \\
\hline$R_{i}$ & Speed drop due to governor action & Hz/p.u.MW \\
\hline $\mathrm{ACE}_{i}$ & Area control error & p.u.MW \\
\hline
\end{tabular}

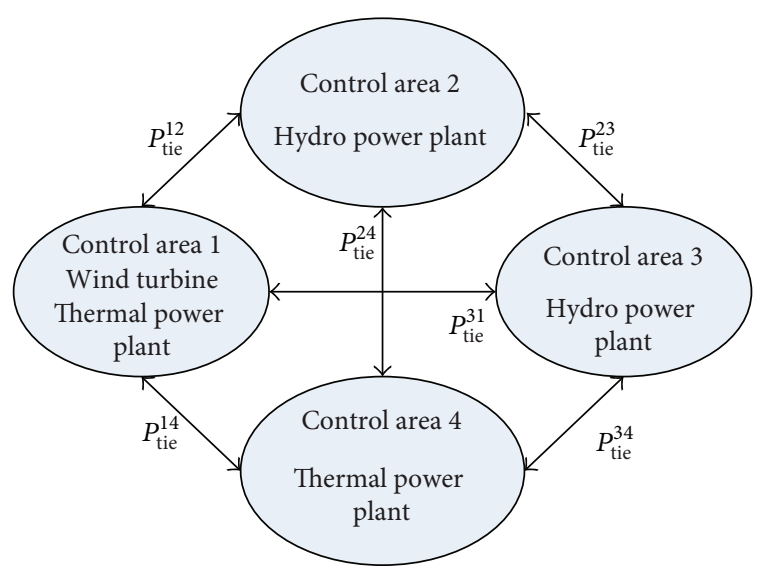

Figure 1: The four-area interconnected hybrid power system.
2.1. Wind Turbine Model. A wind turbine is an installation for converting kinetic energy extracted from wind to electrical energy. Figure 5 illustrates the basic model structure of a wind turbine and the interactions between the different dynamic components in the model. The whole wind turbine can be divided into four subsystems: aerodynamics subsystem, mechanical subsystem, electrical, and actuator subsystem [21].

The linearization model for the variable speed wind turbine in Figure 6 can be represented by

$$
\begin{aligned}
& \Delta \dot{\varphi}_{\varepsilon}=\Delta \omega_{r}-\frac{1}{n_{\text {gear }}} \Delta \omega_{g}, \\
& \Delta \dot{\omega}_{r}=-\frac{K_{s}}{J_{r}} \Delta \varphi_{\varepsilon}+\frac{1}{J_{r}} \Delta T_{r},
\end{aligned}
$$




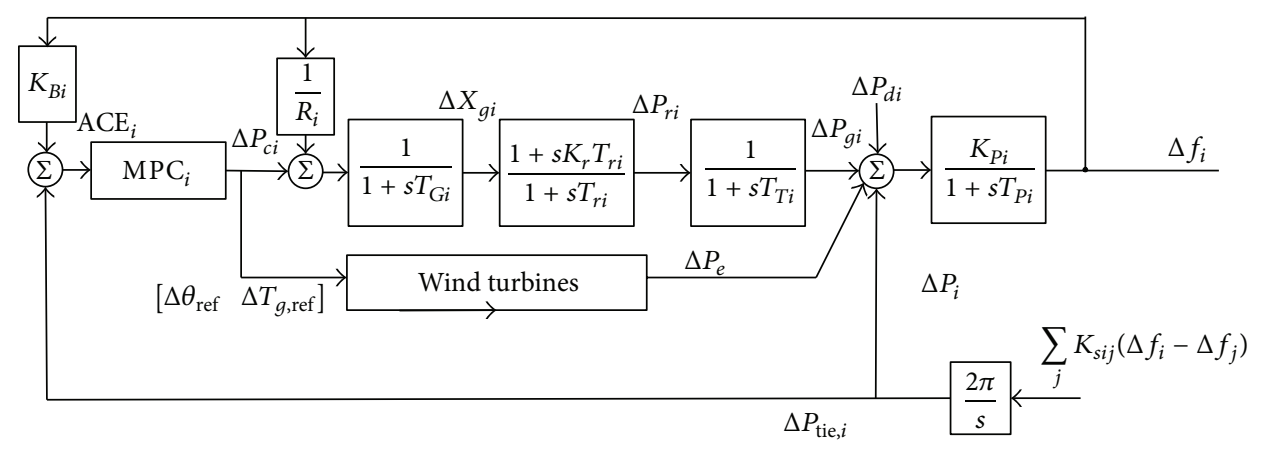

FIGURE 2: Block diagram of a thermal power plant and wind turbines $(i=1)$.

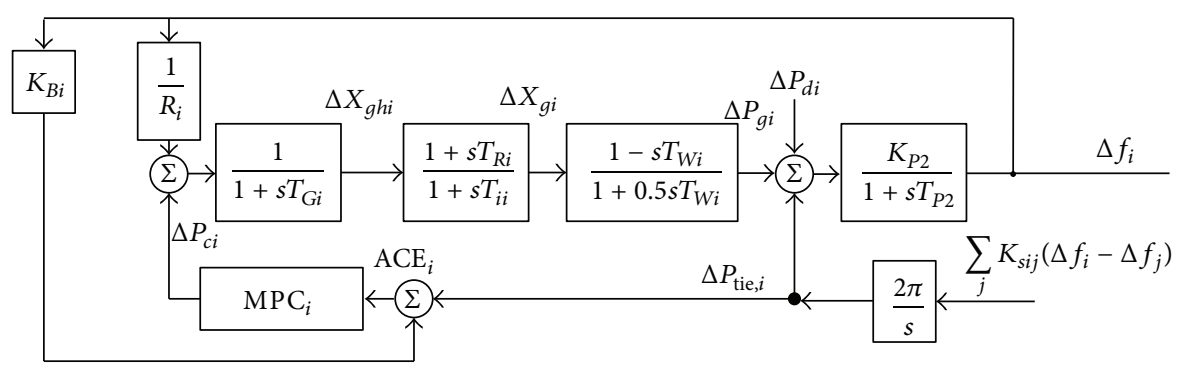

FIgURE 3: Block diagram of a hydro power plant $(i=2,3)$.

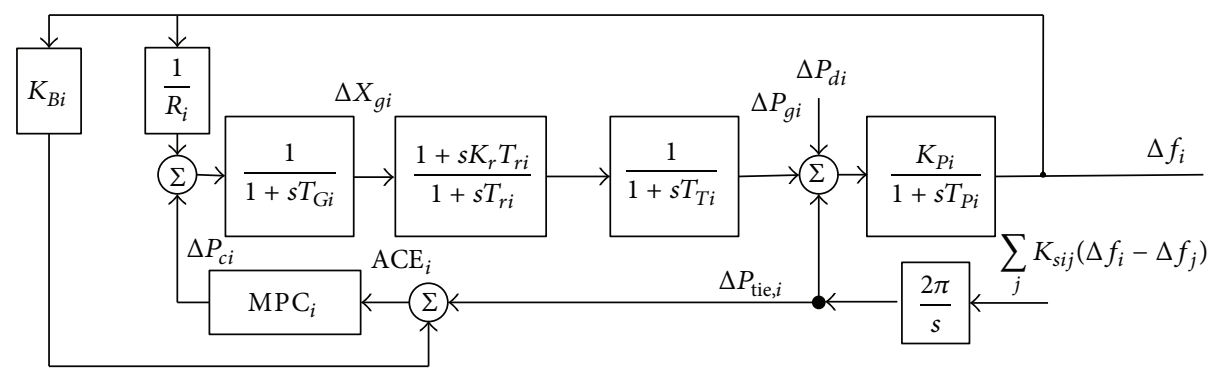

FIGURE 4: Block diagram of a thermal power plant $(i=4)$.

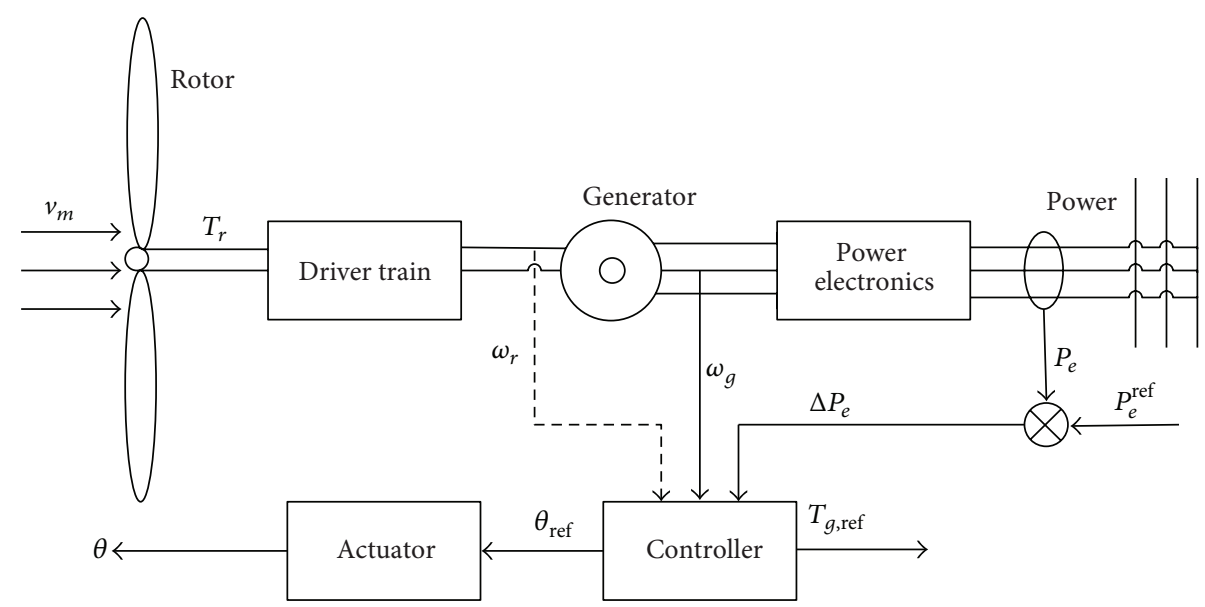

FIGURE 5: Diagram of a variable speed wind turbine. 


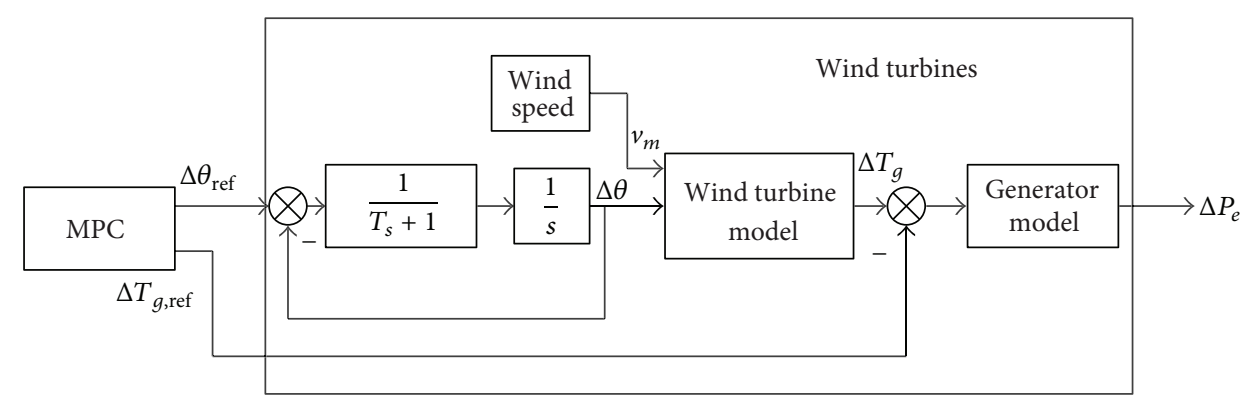

FIgURE 6: Diagram of wind power plant in area 1.

$$
\begin{aligned}
\Delta \dot{\omega}_{g} & =\frac{\eta_{\text {gear }} K_{s}}{n_{\text {gear }}} \Delta \varphi_{\varepsilon}-\frac{1}{J_{r}} \Delta T_{g}, \\
\Delta \dot{\theta} & =-\frac{1}{\tau_{\beta}} \Delta \theta+\frac{K_{\beta}}{\tau_{\beta}} \Delta \theta_{\text {ref }} .
\end{aligned}
$$

The generator reaction torque $T_{g}$ and the reference pitch angle $\theta_{\text {ref }}$ are used as indicator of the input of VSWT, as $u_{e}=\left[\begin{array}{ll}\Delta \beta_{\text {ref }} & \Delta T_{g}\end{array}\right]^{T} \in R^{2}$. Moreover, $\eta$ is the efficiency of the generator and $\omega_{g}$ and $T_{g}$ are used as indicator of the output power as $P_{e}=\eta \omega_{g} T_{g} \in R^{1}$, where $\omega_{g}$ is the angular velocity of generator shaft. A generalized representation of the statespace model of the variable speed turbine can be described as

$$
\begin{aligned}
& \dot{x}_{e}(t)=A\left(v_{m}\right) x_{e}(t)+B_{1}\left(v_{m}\right) \omega(t)+B_{2} u_{e}(t), \\
& z_{e}(t)=C x_{e}(t)+D_{1} \omega(t)+D_{2} u_{e}(t)
\end{aligned}
$$

with

$$
\begin{aligned}
A\left(v_{m}\right) & =\left[\begin{array}{cccc}
0 & 1 & -\frac{1}{n_{\mathrm{gear}}} & 0 \\
-\frac{K_{s}}{J_{r}} & \left.\frac{1}{J_{r}} \frac{\partial T_{r}}{\partial \omega_{r}}\right|_{\mathrm{op}} & 0 & \left.\frac{1}{J_{r}} \frac{\partial T_{r}}{\partial \theta}\right|_{\mathrm{op}} \\
\frac{\eta_{\text {gear }} K_{s}}{n_{\text {gear }} J_{g}} & 0 & 0 & 0 \\
0 & 0 & 0 & -\frac{1}{\tau_{\theta}}
\end{array}\right], \\
B_{1}\left(v_{m}\right) & =\left[\begin{array}{c}
0 \\
\left.\frac{1}{J_{r}} \frac{\partial T_{r}}{\partial v}\right|_{\mathrm{op}} \\
0 \\
0
\end{array}\right], \\
B_{2} & =\left[\begin{array}{cc}
0 & 0 \\
0 & 0 \\
0 & -\frac{1}{J_{g}} \\
K_{\beta} & 0
\end{array}\right],
\end{aligned}
$$

$$
\begin{aligned}
& C=\left[\begin{array}{llll}
0 & 0 & 1 & 0 \\
0 & 0 & 0 & 0
\end{array}\right], \\
& D_{1}=\left[\begin{array}{l}
0 \\
0
\end{array}\right] \text {, } \\
& D_{2}=\left[\begin{array}{ll}
0 & 0 \\
0 & 1
\end{array}\right] \text {, } \\
& x_{e}=\left[\begin{array}{llll}
\Delta \varphi_{\varepsilon} & \Delta \omega_{r} & \Delta \omega_{g} & \Delta \theta
\end{array}\right]^{T} \text {, } \\
& u_{e}=\left[\begin{array}{ll}
\Delta \theta_{\text {ref }} & \Delta T_{g, \text { ref }}
\end{array}\right]^{T} \text {, } \\
& z_{e}=\left[\begin{array}{ll}
\Delta \omega_{g} & \Delta T_{g}
\end{array}\right]^{T}, \\
& y_{e}=P_{e}=\eta \omega_{g} T_{g} .
\end{aligned}
$$

2.2. Four-Area Power System with Wind Turbine. Denoting that the control area $i(i=1,2,3,4)$ is to be interconnected with the control area $j, j \neq i$, through a tie-line, a linear continuous time-varying model of control area $i$ can be written as

$$
\begin{aligned}
& \dot{x}_{i}=A_{i i} x_{i}+B_{i i} u_{i}+F_{i i} d_{i}+\sum_{i \neq j}\left(A_{i j} x_{j}+B_{i j} u_{j}+F_{i j} d_{j}\right), \\
& y_{i}=C_{i i} x_{i}
\end{aligned}
$$

where $x_{i} \in R^{n}, u_{i} \in R^{m}, d_{i} \in R^{k}$, and $y_{i} \in R^{l}$ are the state vector, the control signal vector, the disturbance vector, and the vector of output of control area $i$, respectively. $x_{j} \in R^{p}$, $u_{j} \in R^{q}$, and $d_{j} \in R^{s}$ are the state vector, the control signal vector, and the disturbance vector of neighbor control area, respectively. Matrices $A_{i i}, B_{i i} C_{i i}$, and $F_{i i}$ represent appropriate system matrices of control area $i$, and $A_{i j}, B_{i j}$, and $F_{i j}$ represent the matrices of interaction variables between area $i$ and area $j$. Tie-line power for area $i$ is represented by

$$
\begin{aligned}
\Delta P_{\mathrm{te}, i} & =\sum_{\substack{j=1 \\
j \neq i}}^{4} \Delta P_{\mathrm{tie}}^{i j}=\sum_{\substack{j=1 \\
j \neq i}}^{4} K_{s i j}\left(\Delta f_{i}-\Delta f_{j}\right), \\
\Delta P_{\mathrm{tie}}^{i j} & =-\Delta P_{\mathrm{tie}}^{j i} .
\end{aligned}
$$


The state, disturbance, and output vectors for area $i$ are defined by

$$
\begin{aligned}
& x_{i}=\left[\begin{array}{llllllll}
\Delta f_{i} & \Delta P_{\mathrm{tie}, i} & \Delta P_{g i} & \Delta X_{g i} & \Delta \varphi_{\varepsilon} & \Delta \omega_{r} & \Delta \omega_{g} & \Delta \theta
\end{array}\right]^{T} \\
& (i=1) \text {, } \\
& x_{i}=\left[\begin{array}{llll}
\Delta f_{i} & \Delta P_{\text {tie }, i} & \Delta P_{g i} & \Delta X_{g i} \Delta X_{g h i}
\end{array}\right]^{T} \quad(i=2,3) \text {, } \\
& x_{i}=\left[\begin{array}{lllll}
\Delta f_{i} & \Delta P_{\mathrm{tie}, i} & \Delta P_{g i} & \Delta X_{g i} & \Delta P_{r i}(t)
\end{array}\right]^{T} \quad(i=4),
\end{aligned}
$$

$$
d_{i}=\Delta P_{d i} \quad(i=1,2,3,4),
$$$$
u_{1}=\left[\begin{array}{lll}
\Delta P_{c 1} & \Delta \theta_{\text {ref }} & \Delta T_{g}
\end{array}\right]^{T},
$$$$
y_{i}=\mathrm{ACE}_{i}=\left[K_{B i} \Delta f_{i}+\Delta P_{\mathrm{tie}, i}\right] \quad(i=1,2,3,4) .
$$

The state, control, and disturbance matrices for area 1 are as follows:

$$
\begin{aligned}
& A_{11}=\left[\begin{array}{cccccccc}
-\frac{1}{T_{P 1}} & -\frac{K_{P 1}}{T_{P 1}} & \frac{K_{P 1}}{T_{P 1}} & 0 & 0 & 0 & 0 & 0 \\
\sum_{j} K_{s i j} & 0 & 0 & 0 & 0 & 0 & 0 & 0 \\
0 & 0 & -\frac{1}{T_{T 1}} & 0 & \frac{1}{T_{T 1}} & 0 & 0 & 0 \\
\frac{1}{T_{G 1}} & 0 & 0 & -\frac{1}{T_{G 1}} & 0 & 0 & 0 & 0 \\
0 & 0 & 0 & 0 & 0 & 1 & -\frac{1}{n_{\text {gear }}} & 0 \\
0 & 0 & 0 & 0 & -\frac{K_{s}}{J_{r}} & \left.\frac{1}{J_{r}} \frac{\partial T_{r}}{\partial \omega_{r}}\right|_{\mathrm{op}} & 0 & \frac{1}{J_{r}} \frac{\partial T_{r}}{\partial \theta} \\
0 & 0 & 0 & 0 & \frac{\eta_{\text {gear }}}{n_{\text {gear }} J_{g}} & 0 & 0 & 0 \\
0 & 0 & 0 & 0 & 0 & 0 & 0 & -\frac{1}{\tau_{\theta}}
\end{array}\right] \text {, } \\
& B_{11}=\left[\begin{array}{cccccccc}
0 & 0 & 0 & \frac{1}{T_{G 1}} & 0 & 0 & 0 & 0 \\
0 & 0 & 0 & 0 & 0 & 0 & 0 & \frac{K_{\beta}}{\tau_{\theta}} \\
0 & 0 & 0 & 0 & 0 & 0 & -\frac{1}{J_{g}} & 0
\end{array}\right]^{T} \\
& F_{11}=\left[\begin{array}{cccccccc}
-\frac{K_{P 1}}{T_{P 1}} & 0 & 0 & 0 & 0 & 0 & 0 & 0 \\
0 & 0 & 0 & 0 & 0 & \left.\frac{1}{J_{r}} \frac{\partial T_{r}}{\partial v_{m}}\right|_{\mathrm{op}} & 0 & 0
\end{array}\right]^{T}, \\
& C_{11}=\left[\begin{array}{llllllll}
K_{b 1} & 1 & 0 & 0 & 0 & 0 & 0 & 0
\end{array}\right] \text {. }
\end{aligned}
$$

However for hydro plants in areas 2 and 3 they are as follows:

$$
A_{22}=A_{33}=\left[\begin{array}{ccccc}
-\frac{1}{T_{P i}} & -\frac{K_{P i}}{T_{P i}} & \frac{K_{P i}}{T_{P i}} & 0 & 0 \\
\sum_{j} K_{S i j} & 0 & 0 & 0 & 0 \\
2 \alpha & 0 & -\frac{2}{T_{W i}} & 2 \kappa & 2 \beta \\
-\alpha & 0 & 0 & -\frac{1}{T_{2 i}} & -\beta \\
-\frac{1}{T_{1 i} R_{i}} & 0 & 0 & 0 & -\frac{1}{T_{1 i}}
\end{array}\right] \text {, }
$$

$$
\begin{aligned}
& B_{22}=B_{33}=\left[\begin{array}{lllll}
0 & 0 & -2 R_{i} \alpha & R_{i} \alpha & \frac{1}{T_{1 i}}
\end{array}\right]^{T}, \\
& C_{22}=C_{33}=\left[\begin{array}{lllll}
K_{B i} & 1 & 0 & 0 & 0
\end{array}\right], \\
& F_{22}=F_{33}=\left[\begin{array}{lllll}
-\frac{K_{p i}}{T_{p i}} & 0 & 0 & 0 & 0
\end{array}\right]^{T},
\end{aligned}
$$

where $\alpha=T_{R i} / T_{1 i} T_{2 i} R_{i}, \beta=\left(T_{R i}-T_{1 i}\right) / T_{1 i} T_{2 i}$, and $\kappa=\left(T_{2 i}+\right.$ $\left.T_{W i}\right) / T_{2 i} T_{W i}$. 
However for thermal power plants in area 4 they are as follows:

$$
\begin{aligned}
A_{44} & =\left[\begin{array}{ccccc}
-\frac{1}{T_{P i}} & -\frac{K_{P i}}{T_{P i}} & \frac{K_{P i}}{T_{P i}} & 0 & 0 \\
\sum_{j} K_{S i j} & 0 & 0 & 0 & 0 \\
0 & 0 & -\frac{1}{T_{T i}} & 0 & \frac{1}{T_{T i}} \\
-\frac{1}{T_{G i} R_{i}} & 0 & 0 & -\frac{1}{T_{G i}} & 0 \\
-\frac{K_{r i}}{T_{G i} R_{i}} & 0 & 0 & \frac{1}{T_{r i}}-\frac{K_{r i}}{T_{G i}} & -\frac{1}{T_{r i}}
\end{array}\right], \\
B_{44} & =\left[\begin{array}{llll}
0 & 0 & \frac{1}{T_{G i}} & 0
\end{array}\right]^{T}, \\
C_{44}= & {\left[\begin{array}{llll}
K_{B i} & 1 & 0 & 0
\end{array}\right], } \\
F_{44}= & {\left[\begin{array}{llll}
K_{p i} & 0 & 0 & 0
\end{array}\right]^{T} . }
\end{aligned}
$$

The interaction matrices between the four control areas are as follows:

$$
\begin{aligned}
& A_{i j}=\left[\begin{array}{cccccccc}
0 & 0 & 0 & 0 & 0 & 0 & 0 & 0 \\
-K_{S i j} & 0 & 0 & 0 & 0 & 0 & 0 & 0 \\
0 & 0 & 0 & 0 & 0 & 0 & 0 & 0 \\
0 & 0 & 0 & 0 & 0 & 0 & 0 & 0 \\
0 & 0 & 0 & 0 & 0 & 0 & 0 & 0
\end{array}\right] \\
& (i=1, j=2,3,4), \\
& A_{i j}=\left[\begin{array}{cccccccc}
0 & 0 & 0 & 0 & 0 & 0 & 0 & 0 \\
-K_{S i j} & 0 & 0 & 0 & 0 & 0 & 0 & 0 \\
0 & 0 & 0 & 0 & 0 & 0 & 0 & 0 \\
0 & 0 & 0 & 0 & 0 & 0 & 0 & 0
\end{array}\right] \\
& (i=1, j=2,3, i \neq j), \\
& A_{i j}=\left[\begin{array}{ccccc}
0 & 0 & 0 & 0 & 0 \\
-K_{S i j} & 0 & 0 & 0 & 0 \\
0 & 0 & 0 & 0 & 0 \\
0 & 0 & 0 & 0 & 0
\end{array}\right] \quad(i=j=2,3,4, i \neq j) \text {, } \\
& B_{i j}=0_{8 \times 4}, \\
& F_{i j}=0_{8 \times 2} \\
& (i=1, j=2,3,4, \quad i \neq j),
\end{aligned}
$$

$$
\begin{gathered}
B_{i j}=0_{5 \times 1}, \\
F_{i j}=0_{5 \times 1}
\end{gathered}
$$

$$
(i=j=2,3,4, i \neq j) \text {. }
$$

The GRCs for the thermal plants are $\left|\Delta \dot{P}_{g i}\right| \leq 0.0017$ p.u.MW/ $s$ and the hydro units are $\left|\Delta \dot{P}_{g i}\right| \leq 0.045$ p.u.MW/s. In addition, the load disturbance is constrained to $\left|\Delta \dot{P}_{d_{i}}\right| \leq 0.3$.

\section{Distributed Model Predictive Controller}

3.1. Distributed Model Predictive Controller. The block diagram of the DMPC scheme for a four-area interconnected power system is illustrated in Figure 7. Though there exists large amount of variables in the interconnected power system, the 30 state variables expressed in (1a), (1b), (1c), and (1d) concerning the frequency, the generator output power, the governor valve (servomotor) position, the tie-line active power, the wind power, and the 4 load disturbance $\Delta P_{d i}$ are crucial to LFC problem. They can be measured or estimated directly by the local controller. The DMPC in each area exchange control information through the power line communication, which is a sole networking technology with high reliability that can provide high speed communication to power grids applications [22].

Distributed MPC. The partitioned discrete-time model for control area $i$ of the continuous-time four-area interconnected power system ((1a), (1b), (1c), and (1d)) can be expressed as follows:

$$
\begin{aligned}
x_{i}(k+1)= & \bar{A}_{i i} x_{i}(k)+\bar{B}_{i i} u_{i}(k)+\bar{F}_{i i} d_{i}(k) \\
& +\sum_{i \neq j}\left(\bar{A}_{i j} x_{j}(k)+\bar{B}_{i j} u_{j}(k)+\bar{F}_{i j} d_{j}(k)\right), \\
y_{i}(k)= & \bar{C}_{i i} x_{i}(k),
\end{aligned}
$$

where $\bar{A}_{i i}, \bar{B}_{i i}, \bar{C}_{i i}, \bar{F}_{i i}, \bar{A}_{i j}, \bar{B}_{i j}$, and $\bar{F}_{i j}$ represent the discrete new matrices obtained from original matrices in (4) based on the Zero-Order Hold $(\mathrm{ZOH})$ method.

Assume that the state variables $x_{i}(k)$ and the disturbance $D_{i}$ can be measured or estimated directly by the controller in area $i$ at sampling time $k$. Optimizations and exchange of variables are termed iterate. The iteration number is denoted by $p$.

For DMPC, the optimal state-input trajectory $\left(x_{i}, u_{i}\right)$ for each area $i, i=1,2,3,4$ at iterate $p$ is obtained as the solution to the optimization problem:

$$
\begin{array}{ll}
\min _{u_{i}(k+n \mid k)} & J_{i}(k) \\
& J_{i}(k)=\sum_{n=0}^{N}\left[x_{i}^{T}(k+n \mid k) Q_{i} x_{i}(k+n \mid k)+u_{i}^{T}(k+n \mid k) R_{i} u_{i}(k+n \mid k)\right]
\end{array}
$$




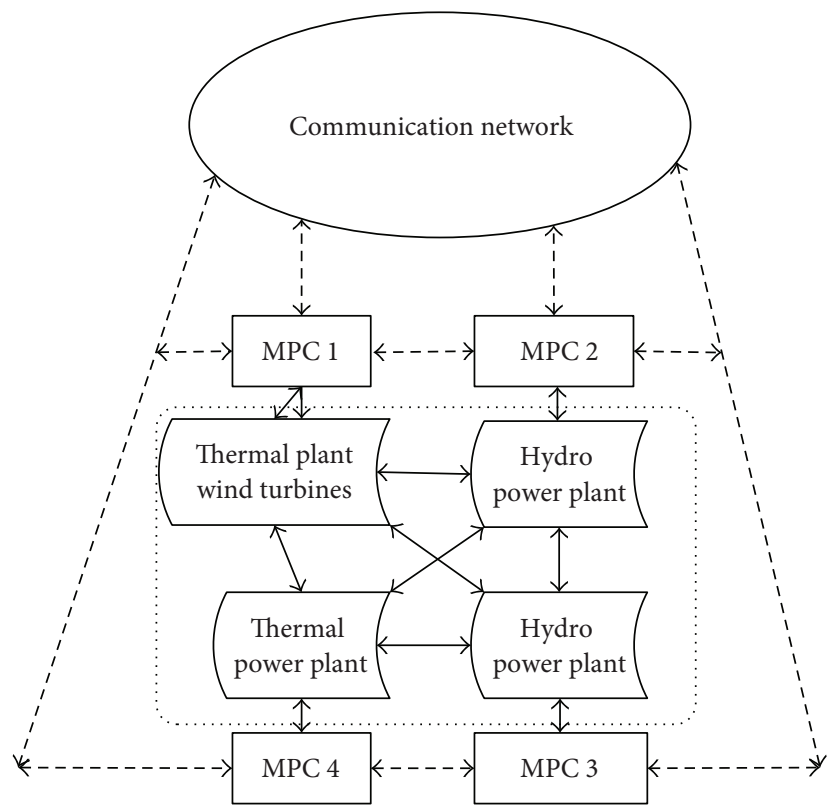

FIGURE 7: Block diagram of DMPC for power system with wind turbines.

$$
\begin{array}{ll}
\text { Subject to } & \left\|x_{i 3}(k+n \mid k)\right\|_{2} \leq 0.0017, \quad i=1,4 \\
& \left\|x_{i 3}(k+n \mid k)\right\|_{2} \leq 0.0045, \quad i=2,3 \\
& \left\|x_{i 4}(k+n \mid k)\right\|_{2} \leq \sigma_{i}, \quad i=1,2,3,4 .
\end{array}
$$

For notational convenience, we drop the $k$ dependence of $x_{i}(k), u_{i}(k), i=1,2,3,4$. It is shown in [20] that each $\bar{x}_{i}$ can be expressed as

$$
\begin{aligned}
\bar{x}_{i}= & \bar{E}_{i i} \bar{u}_{i}+\bar{f}_{i i} x_{i}(k)+\bar{\beta}_{i i} d_{i}(k) \\
& +\sum_{i \neq j}\left(\bar{E}_{i j} \bar{u}_{j}+\bar{g}_{i j} \bar{x}_{j}+\bar{f}_{i j} x_{j}(k)+\bar{\beta}_{i j} d_{j}(k)\right)
\end{aligned}
$$

with

$\bar{x}_{i}$

$$
=\left[\begin{array}{llll}
x_{i}(k+1 \mid k)^{T} & x_{i}(k+2 \mid k)^{T} & \cdots & x_{i}\left(k+N_{p} \mid k\right)^{T}
\end{array}\right]^{T},
$$

$\bar{u}_{i}$

$$
=\left[\begin{array}{llll}
u_{i}(k \mid k)^{T} & u_{i}(k+1 \mid k)^{T} & \cdots & u_{i}\left(k+N_{c}-1 \mid k\right)^{T}
\end{array}\right]^{T} .
$$

Let $N_{c}$ denote the control horizon and let $N_{p}$ denote the predictive horizon. $\bar{x}_{i}$ is no more a vector but a matrix after iteration obtained from original equation (4). The matrices in (15) have detailed expressions as follows:

$$
\bar{E}_{i i}=\left[\begin{array}{cccc}
\bar{B}_{i i} & 0 & \cdots & 0 \\
\bar{A}_{i i} \bar{B}_{i i} & \bar{B}_{i i} & \cdots & 0 \\
\vdots & \vdots & & \vdots \\
\bar{A}_{i i}^{N-1} \bar{B}_{i i} & \bar{A}_{i i}^{N-2} & \ldots & 0
\end{array}\right] \text {, }
$$

$$
\bar{E}_{i j}=\left[\begin{array}{cccc}
\bar{B}_{i j} & 0 & \cdots & 0 \\
\bar{A}_{i i} \bar{B}_{i j} & \bar{B}_{i j} & \cdots & 0 \\
\vdots & \vdots & & \vdots \\
\bar{A}_{i i}^{N-1} \bar{B}_{i j} & \bar{A}_{i i}^{N-2} & \cdots & 0
\end{array}\right] \text {, }
$$

$$
\bar{f}_{i i}=\left[\begin{array}{c}
\bar{A}_{i i} \\
\bar{A}_{i i} \bar{A}_{i i} \\
\vdots \\
\bar{A}_{i i}^{N-1} \bar{A}_{i i}
\end{array}\right],
$$




$$
\begin{array}{cc}
\bar{f}_{i j}=\left[\begin{array}{c}
\bar{A}_{i j} \\
\bar{A}_{i i} \bar{A}_{i j} \\
\vdots \\
\bar{A}_{i i}^{N-1} \bar{A}_{i j}
\end{array}\right], & \phi=\left[\begin{array}{llll}
\bar{\beta}_{11} & \bar{\beta}_{12} & \bar{\beta}_{13} & \bar{\beta}_{14} \\
\bar{\beta}_{21} & \bar{\beta}_{22} & \bar{\beta}_{23} & \bar{\beta}_{24} \\
\bar{\beta}_{31} & \bar{\beta}_{32} & \bar{\beta}_{33} & \bar{\beta}_{34} \\
\bar{\beta}_{41} & \bar{\beta}_{42} & \bar{\beta}_{43} & \bar{\beta}_{44}
\end{array}\right], \\
\bar{\beta}_{i i} \\
\bar{\beta}_{i i}=\left[\begin{array}{c}
\bar{A}_{i i} \bar{F}_{i i} \\
\vdots
\end{array}\right], & \tilde{x}=\left[\begin{array}{llll}
\bar{x}_{1} & \bar{x}_{2} & \bar{x}_{3} & \bar{x}_{4}
\end{array}\right]^{T}, \\
\end{array}
$$$$
\bar{\beta}_{i i}=\left[\begin{array}{c}
\bar{F}_{i i} \\
\bar{A}_{i i} \bar{F}_{i i} \\
\vdots \\
\bar{A}_{i i}^{N-1} \bar{F}_{i i}
\end{array}\right],
$$$$
\bar{\beta}_{i j}=\left[\begin{array}{c}
\bar{F}_{i j} \\
\bar{A}_{i i} \bar{F}_{i j} \\
\vdots \\
\bar{A}_{i i}^{N-1} \bar{F}_{i j}
\end{array}\right] \text {, }
$$$$
\bar{g}_{i j}=\left[\begin{array}{ccccc}
0 & 0 & 0 & \cdots & 0 \\
\bar{A}_{i j} & 0 & 0 & \cdots & 0 \\
\bar{A}_{i i} \bar{A}_{i j} & \bar{A}_{i j} & 0 & \cdots & 0 \\
\vdots & \vdots & \vdots & \cdots & \vdots \\
\bar{A}_{i i}^{N-2} \bar{A}_{i j} & \bar{A}_{i i}^{N-3} \bar{A}_{i j} & \ldots & \bar{A}_{i j} & 0
\end{array}\right] \text {, }
$$

Since matrix $\Lambda$ is invertible, we can write it as

$$
\begin{aligned}
\bar{x}_{i}= & \bar{E}_{i i} \bar{u}_{i}+\bar{f}_{i i} x_{i}(k)+\bar{\beta}_{i i} d_{i}(k) \\
& +\sum_{i \neq j}\left(\bar{E}_{i j} \bar{u}_{j}+\bar{f}_{i j} x_{j}(k)+\bar{\beta}_{i j} d_{j}(k)\right)
\end{aligned}
$$

in which

$$
\begin{aligned}
& \bar{E}_{i j}=\Lambda^{-1} \varepsilon, \\
& \bar{f}_{i j}=\Lambda^{-1} \mu, \\
& \bar{\beta}_{i j}=\Lambda^{-1} \phi .
\end{aligned}
$$

where $\bar{E}_{i i}, \bar{f}_{i i}, \bar{\beta}_{i i}, \bar{E}_{i j}, \bar{f}_{i j}, \bar{\beta}_{i j}$, and $\bar{g}_{i j}$ are the new matrices obtained from $\bar{A}_{i i}, \bar{B}_{i i}, \bar{C}_{i i}, \bar{F}_{i i}, \bar{A}_{i j}, \bar{B}_{i j}$, and $\bar{F}_{i j}$ after iteration.

Combining the models in (15) gives the following system of equations:

$$
\Lambda \tilde{x}=\varepsilon \tilde{u}+\mu \tilde{x}(k)+\phi d(k)
$$

with

$$
\begin{aligned}
& \Lambda=\left[\begin{array}{cccc}
I & -\bar{g}_{12} & -\bar{g}_{13} & -\bar{g}_{14} \\
-\bar{g}_{21} & I & -\bar{g}_{23} & -\bar{g}_{24} \\
-\bar{g}_{31} & -\bar{g}_{32} & I & -\bar{g}_{34} \\
-\bar{g}_{41} & -\bar{g}_{42} & -\bar{g}_{43} & I
\end{array}\right], \\
& \varepsilon=\left[\begin{array}{llll}
\bar{E}_{11} & \bar{E}_{12} & \bar{E}_{13} & \bar{E}_{14} \\
\bar{E}_{21} & \bar{E}_{22} & \bar{E}_{23} & \bar{E}_{24} \\
\bar{E}_{31} & \bar{E}_{32} & \bar{E}_{33} & \bar{E}_{34} \\
\bar{E}_{41} & \bar{E}_{42} & \bar{E}_{43} & \bar{E}_{44}
\end{array}\right] \text {, } \\
& \mu=\left[\begin{array}{llll}
\bar{f}_{11} & \bar{f}_{12} & \bar{f}_{13} & \bar{f}_{14} \\
\bar{f}_{21} & \bar{f}_{22} & \bar{f}_{23} & \bar{f}_{24} \\
\bar{f}_{31} & \bar{f}_{32} & \bar{f}_{33} & \bar{f}_{34} \\
\bar{f}_{41} & \bar{f}_{42} & \bar{f}_{43} & \bar{f}_{44}
\end{array}\right],
\end{aligned}
$$

To do so, we eliminate the unknown matrix $\bar{x}_{j}$ because we have knowledge of $x_{j}(k)$ since it is just a vector at time $k$.

In the distributed MPC algorithm, for subsystem $i$, the control signal $\bar{U}_{i}$ is designed at each time interval $k \geq 0$. By solving the following optimization problem denoted by $J_{i}$, it is usually defined as

$$
J_{i}=\min _{\bar{u}_{i}} \frac{1}{2} \bar{u}_{i}^{T} \Phi_{i} \bar{u}_{i}^{T}+\left(\gamma_{i}+\Gamma_{i}+\sum_{i \neq j} H_{i j} \bar{u}_{j}\right)^{T} \bar{u}_{i}
$$

in which

$$
\begin{aligned}
& \mathbb{Q}_{i}=\operatorname{diag} \overbrace{\left(\omega_{i} Q_{i}, \ldots, \omega_{i} Q_{i}\right)}^{N_{p}}, \\
& \mathbb{R}_{i}=\operatorname{diag} \overbrace{\left(\omega_{i} R_{i}, \ldots, \omega_{i} R_{i}\right)}^{N_{c}}, \\
& \Phi_{i}=\mathbb{R}_{i}+\bar{E}_{i i}^{T} \mathbb{Q}_{i} \bar{E}_{i i}+\sum_{\substack{j=1 \\
j \neq i}}^{4} \bar{E}_{j i}^{T} \mathbb{Q}_{j} \bar{E}_{j i}, \\
& \gamma_{i}=\bar{E}_{i i}^{T} \mathbb{Q}_{i} \bar{g}_{i i}+\sum_{\substack{j=1 \\
j \neq i}}^{4} \bar{E}_{j i}^{T} \mathbb{Q}_{j} \bar{g}_{j i},
\end{aligned}
$$




$$
\begin{gathered}
g_{i i}=f_{i i} x_{i}(k)+\sum_{j=1}^{4} f_{i j} x_{j}(k), \\
\Gamma_{i}=E_{i i}^{T} \mathbb{Q}_{i} \rho_{i}+\sum_{j=1}^{4} E_{j i}^{T} \mathbb{Q}_{j} \rho_{j}, \\
\rho_{i}=\beta_{i i} d_{i}(k)+\sum_{j=1}^{4} \beta_{i j} d_{j}(k), \\
H_{i j}=\bar{E}_{i i}^{T} \mathbb{Q}_{i} \bar{E}_{i j}+\sum_{\substack{j=1 \\
j \neq i}}^{4} \bar{E}_{j i}^{T} \mathbb{Q}_{j} \bar{E}_{j i} .
\end{gathered}
$$

At time interval $k,(22)$ is implemented based on the future states and manipulated variables. The first input in the optimal sequence is injected into the processes, and the procedure is repeated at subsequent time intervals.

$Q_{i} \geq 0, R_{i} \geq 0$ are symmetric weighting matrices and $\omega_{i}>0, \sum_{i=1}^{4} \omega_{i}=1$.

Define $\eta_{i}=\gamma_{i}+\Gamma_{i}+\sum_{j \neq i} H_{i j} \bar{u}_{j}$.

Then (22) is rewritten as

$$
J_{i}=\min _{\bar{u}_{i}} \frac{1}{2} \bar{u}_{i}^{T} \Phi_{i} u_{i}^{T}+\eta_{i}^{T} \bar{u}_{i}
$$

3.2. Constraint Handling. The two crucial nonlinearities, for example, the GRCs and the valve position limits of the governor, have been considered as the state constraints in the designed DMPC, as shown in Figures 8 and 9.
In power system, the GRC can be expressed as $\Delta \dot{P}_{g}(k)_{\min } \leq \Delta \dot{P}_{g}(k) \leq \Delta \dot{P}_{g}(k)_{\max }$, and then the constraints on $\Delta P_{g}$ can be expressed as follows:

$$
\begin{aligned}
& T\left(\Delta \dot{P}_{g}(k)\right)_{\min }+\Delta P_{g}(k-1) \leq \Delta P_{g}(k) \\
& \leq T\left(\Delta \dot{P}_{g}(k)\right)_{\max }+\Delta P_{g}(k-1), \\
& \Delta \bar{P}_{g} \\
& =\left[\begin{array}{lllll}
\Delta P_{g}(k+1 \mid k) & \Delta P_{g}(k+2 \mid k) & \cdots & \Delta P_{g}\left(k+N_{p} \mid k\right)
\end{array}\right]^{T} .
\end{aligned}
$$

Since $\Delta P_{g i}=X_{i 3}$, the state form can be expressed as

$$
\Delta \bar{P}_{g}=S_{i} \bar{x}_{i}
$$

where $S_{i}=\operatorname{diag}(\overbrace{\omega_{i} S_{i i}, \ldots, \omega_{i} S_{i i}}^{N_{p}})$.

When $i=1,4, S_{i i}=\left[\begin{array}{lllll}0 & 0 & 1 & 0 & 0\end{array}\right]$, and when $i=2,3$, $S_{i i}=\left[\begin{array}{lllll}0 & 0 & 1 & 0 & 0\end{array}\right]$, with (25) and (27), the constraints on $\Delta P_{g}(k)$ are expressed as $\bar{N}_{i} \leq S_{i} \bar{x}_{i} \leq \bar{M}_{i}$.

Define

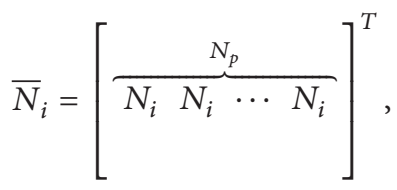

$$
\begin{aligned}
& \bar{M}_{i}=[\overbrace{M_{i} M_{i} \cdots M_{i}}^{N_{p}}]^{T} \text {, }
\end{aligned}
$$

where $N_{i}$ and $M_{i}$ are obtained from (15).

Consider the constraints on $\Delta P_{g}(k)$ :

$$
\left[\begin{array}{c}
S_{i} E_{i i} \\
-S_{i} E_{i i}
\end{array}\right] \bar{u}_{i} \leq\left[\begin{array}{c}
\bar{M}_{i}-S_{i}\left\{f_{i i} x_{i}(k)+\beta_{i i} d_{i}(k)+\sum_{j \neq i}\left[E_{i j} \bar{u}_{j}+f_{i j} x_{j}(k)+\beta_{i j} d_{j}(k)\right]\right\} \\
-\bar{N}_{i}+S_{i}\left\{f_{i i} x_{i}(k)+\beta_{i i} d_{i}(k)+\sum_{i \neq j}\left[E_{i j} \bar{u}_{j}+f_{i j} x_{j}(k)+\beta_{i j} d_{j}(k)\right]\right\}
\end{array}\right] .
$$

Define

$$
\begin{aligned}
\Psi_{i} & =\left[\begin{array}{c}
S_{i} E_{i i} \\
-S_{i} E_{i i}
\end{array}\right], \\
\Pi_{i} & =\left[\begin{array}{l}
\bar{M}_{i}-S_{i}\left\{f_{i i} x_{i}(k)+\beta_{i i} d_{i}(k)+\sum_{j \neq i}\left[E_{i j} \bar{u}_{j}+f_{i j} x_{j}(k)+\beta_{i j} d_{j}(k)\right]\right\} \\
-\bar{N}_{i}+S_{i}\left\{f_{i i} x_{i}(k)+\beta_{i i} d_{i}(k)+\sum_{i \neq j}\left[E_{i j} \bar{u}_{j}+f_{i j} x_{j}(k)+\beta_{i j} d_{j}(k)\right]\right\}
\end{array}\right] .
\end{aligned}
$$




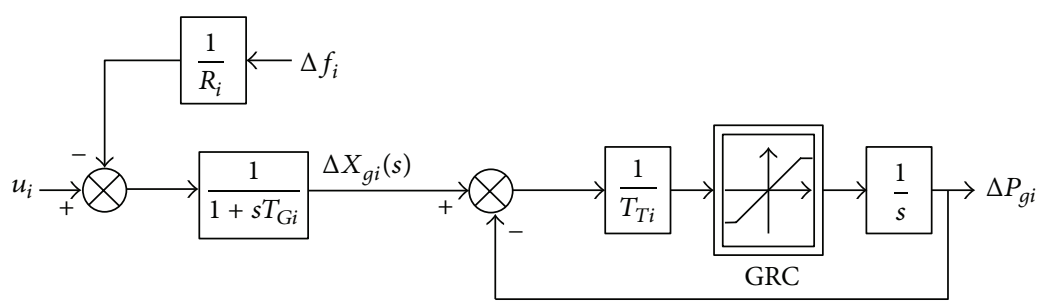

FIgURE 8: Thermal power plant with GRC.

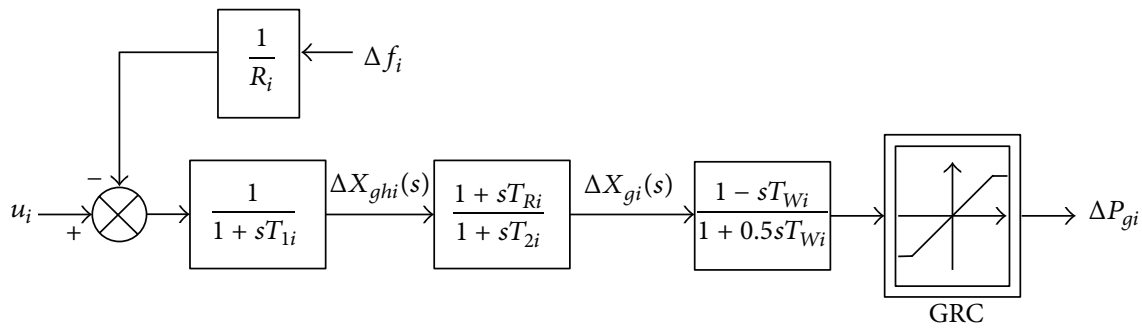

FIGURE 9: Hydro power plant with GRC.

Then, distributed MPC algorithm (24) for multiple-interconnected system can be transformed into the following optimization problem with GRC constraints:

$$
\begin{array}{ll}
J_{i}=\min _{\bar{u}_{i}} & \frac{1}{2} \tilde{u}_{i}^{T} \Phi_{i} \bar{u}_{i}^{T}+\eta_{i}^{T} \bar{u}_{i} \\
\text { Subject to } & \Psi_{i} \bar{u}_{i} \leq \Pi_{i} .
\end{array}
$$

\subsection{The DMPC Algorithm}

Step 1 (initialization). The constant matrices $R_{i}, R_{j}$ and $Q_{i}$, $Q_{j}$, at control interval $k=0$, are given. Choose the specified error tolerance $\varepsilon_{i}$. Set iteration $p=0$.

Step 2 (communication). The controller in each subsystem $i$ exchanges its previous predictions $\bar{x}_{i}(k), \bar{x}_{j}(k)$, set $\bar{u}_{i}^{0}(k)$, and $\bar{u}_{j}^{0}(k)$ at initial instant.

Step 3 (optimization and iteration).

While $p<p_{\text {max }}$,

$\bar{u}_{i}^{*(p)}$ is solved by the optimal problem (31)

$$
\text { If }\left\|u_{i}^{(p)}-u_{i}^{(p-1)}\right\| \leq \varepsilon_{i} \forall i \in\{1,2,3,4\}
$$

Break

End if

Exchange the solutions $\bar{u}_{i}^{p}$ and $\bar{u}_{j}^{p}$ and set $p=p+1$

$$
\text { If } \varepsilon_{i}=0 \forall i \in\{1,2,3,4\}
$$

Break

End if

End while
Step 4 (assignment and prediction). Send out $u_{i}(k)=\bar{u}_{i}(k)$. Otherwise, $u_{i}(k)=\bar{u}_{i}(k-1)$. Predict the future states.

Step 5 (implementation). Set $k=k+1$, and repeat Step 1 .

\section{Simulation Results}

In this section, the four-area power system stability is analyzed, and the performances of the proposed DMPC have been tested in case of wind turbines participation at nominal parameters. The simulation of the proposed DMPC scheme is also verified by two cases. The performance and the implementation of the proposed DMPC are compared with other two types of typical LFC scheme.

As comparison, we design the centralized MPC and decentralized MPC controller for four-area interconnected power system, respectively. The four-area interconnected power system can be described as

$$
\begin{aligned}
& x(k+1)=A x(k)+B u(k)+F d(k), \\
& y(k+1)=C x(k),
\end{aligned}
$$

where

$$
\begin{gathered}
A=\left[\begin{array}{llll}
A_{11} & A_{12} & A_{13} & A_{14} \\
A_{21} & A_{22} & A_{23} & A_{24} \\
A_{31} & A_{32} & A_{33} & A_{34} \\
A_{41} & A_{42} & A_{43} & A_{44}
\end{array}\right], \\
B=\left[\begin{array}{llll}
B_{11} & B_{12} & B_{13} & B_{14} \\
B_{21} & B_{22} & B_{23} & B_{24} \\
B_{31} & B_{32} & B_{33} & B_{34} \\
B_{41} & B_{42} & B_{43} & B_{44}
\end{array}\right],
\end{gathered}
$$




$$
\begin{aligned}
& C=\left[\begin{array}{cccc}
C_{11} & 0 & 0 & 0 \\
0 & C_{22} & 0 & 0 \\
0 & 0 & C_{33} & 0 \\
0 & 0 & 0 & C_{44}
\end{array}\right], \\
& F=\left[\begin{array}{cccc}
F_{11} & 0 & 0 & 0 \\
0 & F_{22} & 0 & 0 \\
0 & 0 & F_{33} & 0 \\
0 & 0 & 0 & F_{44}
\end{array}\right], \\
& x=\left[\begin{array}{llll}
x_{1}^{T} & x_{2}^{T} & x_{3}^{T} & x_{4}^{T}
\end{array}\right]^{T}, \\
& u=\left[\begin{array}{llll}
u_{1}^{T} & u_{2}^{T} & u_{3}^{T} & u_{4}^{T}
\end{array}\right]^{T}, \\
& y=\left[\begin{array}{llll}
y_{1}^{T} & y_{2}^{T} & y_{3}^{T} & y_{4}^{T}
\end{array}\right]^{T}, \\
& d=\left[\begin{array}{llll}
d_{1}^{T} & d_{2}^{T} & d_{3}^{T} & d_{4}^{T}
\end{array}\right]^{T}
\end{aligned}
$$

with constraints (12), (13), (14a), (14b), and (14c) for each control area. In centralized MPC framework, the MPC for overall system (32) solves the following optimization problem:

$$
\begin{aligned}
& \min _{u(k+n \mid k)} J(k) \\
& J(k)=\sum_{n=0}^{N}\left[x^{T}(k+n \mid k) Q x(k+n \mid k)\right. \\
& \left.\quad+u^{T}(k+n \mid k) R u(k+n \mid k)\right]
\end{aligned}
$$

subject to (14a), (14b), and (14c).

The weighting matrices $Q$ and $R$ in objective function (35) are chosen as $R=\operatorname{diag}(1,1,1,1)$ and

$$
Q=\operatorname{diag}\left(\begin{array}{c}
1000,0,0,1000,1000,0,0,1000,1000, \\
0,0,1000,1000,0,0,1000
\end{array}\right) .
$$

In the decentralized modeling framework, it is assumed that the interaction between the control areas is negligible. Subsequently, the decentralized model for each control area is

$$
\begin{aligned}
& x_{i}(k+1)=A_{i i} x_{i}(k)+B_{i i} u_{i}(k)+F_{i i} d_{i}(k), \\
& y_{i}(k+1)=C_{i i} x_{i}(k)
\end{aligned}
$$

with the system matrices and constraints (12), (13), (14a), (14b), and (14c) for each control area denoted as in Section 2. In decentralized MPC framework, each control area based MPC solves the following optimization problem:

$$
\begin{aligned}
& \min _{u_{i}(k+n \mid k)} J_{i}(k) \\
& J_{i}(k)=\sum_{n=0}^{N}\left[x_{i}^{T}(k+n \mid k) Q_{i} x_{i}(k+n \mid k)\right. \\
& \left.\quad+u_{i}^{T}(k+n \mid k) R_{i} u_{i}(k+n \mid k)\right]
\end{aligned}
$$

subject to (14a), (14b), and (14c).
The weighting matrices $Q_{i}$ and $R_{i}$ in objective function (39) are chosen as $R_{1}=R_{2}=R_{3}=R_{4}=1$ and

$$
Q_{1}=Q_{2}=Q_{3}=Q_{4}=\operatorname{diag}(1000,0,0,1000) .
$$

Choose the prediction horizon of the centralized MPC, decentralized MPC, and RDMPC to be $N=15$, choose the control horizon to be $N_{c}=10$, and choose the sample time $T_{s}=0.1$ and $\lambda=0.1$. Consider GRC for the thermal power plants in area 1 and area 4 to be $\left|\Delta \dot{P}_{g}^{i}\right| \leq r=$ 0.1 p.u.MW $/ \mathrm{min}=0.0017$ p.u.MW/s and GRC for the hydro power plants in area 2 and area 3 to be $\left|\Delta \dot{P}_{g}^{i}\right| \leq r=$ 2.7 p.u.MW $/ \mathrm{min}=0.045$ p.u.MW/s. In addition, area 1 includes an aggregated wind turbine model which consists of 30 wind turbine units of $2 \mathrm{MW}$ rated VSWTs while the capacity of thermal plant is $600 \mathrm{MW}$. The wind turbine parameters and operating points [23] are indicated as follows:

Operating point: $80 \mathrm{MW}$; wind speed: $12 \mathrm{~m} / \mathrm{s}$.

$T_{g}=3781.9 \mathrm{Nm} ; \omega_{g}=105 \mathrm{rad} / \mathrm{s} ; \omega_{r}=2.6869 \mathrm{rad} / \mathrm{s}$.

$K_{s}=7.87 e 6 \mathrm{Nm} / \mathrm{rad} ; n_{\text {gear }}=1: 28.7 ; \eta_{\text {gear }}=97.5 \%$.

$J_{r}=2.8675 \mathrm{kgm}^{2} ; J_{g}=54.5432 \mathrm{kgm}^{2}$.

$R_{3}=3.3 \mathrm{~Hz} /$ p.u.MW; $R_{4}=3 \mathrm{~Hz} /$ p.u.MW.

The parameters for the thermal and hydro plants used in the simulation are listed as follows:

$$
\begin{aligned}
& K_{P 1}=120 \mathrm{~Hz} / \text { p.u.MW; } K_{P 2}=115 \mathrm{~Hz} / \text { p.u.MW } \\
& K_{P 3}=80 \mathrm{~Hz} / \text { p.u.MW; } K_{P 4}=75 \mathrm{~Hz} / \text { p.u.MW. } \\
& T_{P 1}=20 \mathrm{~s} ; T_{P 2}=20 \mathrm{~s} ; T_{P 3}=13 \mathrm{~s} ; T_{P 4}=15 \mathrm{~s} . \\
& R_{1}=2.4 \mathrm{~Hz} / \text { p.u.MW; } R_{2}=2.5 \mathrm{~Hz} / \text { p.u.MW. } \\
& R_{3}=3.3 \mathrm{~Hz} / \text { p.u.MW; } R_{4}=3 \mathrm{~Hz} / \text { p.u.MW. } \\
& K_{B 1}=0.425 \text { p.u.MW/Hz; } K_{B 2}=0.409 \text { p.u.MW } / \mathrm{Hz} . \\
& K_{B 3}=0.316 \text { p.u.MW/Hz; } K_{B 4}=0.347 \text { p.u.MW } / \mathrm{Hz} . \\
& T_{G 1}=0.08 \mathrm{~s} ; T_{G 2}=0.1 \mathrm{~s} ; T_{G 3}=0.08 \mathrm{~s} ; T_{G 4}=0.2 \mathrm{~s} . \\
& T_{T 1}=T_{T 4}=0.3 \mathrm{~s} ; T_{r 1}=T_{r 4}=10 \mathrm{~s} ; T_{R 2}=0.6 \mathrm{~s} . \\
& T_{R 3}=0.513 \mathrm{~s} ; T_{22}=5 \mathrm{~s} ; T_{23}=10 \mathrm{~s} ; T_{W 2}=1 \mathrm{~s} ; T_{W 3}= \\
& 2 \mathrm{~s} . \\
& K_{S 12}=-K_{S 21}=0.545 \text { p.u.MW. } \\
& K_{S 23}=-K_{S 32}=0.444 \text { p.u.MW. } \\
& K_{S 13}=-K_{S 31}=0.545 \text { p.u.MW. } \\
& K_{S 14}=-K_{S 41}=0.5 \text { p.u.MW. } \\
& K_{S 24}=-K_{S 42}=0.545 \text { p.u.MW } \\
& K_{S 34}=-K_{S 43}=0.545 \text { p.u.MW. }
\end{aligned}
$$

Case 1 (response to step load change without wind turbines participation). Wind turbine is present but it does not provide any power support in the event of grid frequency deviation. An event is simulated in which a system shown in 

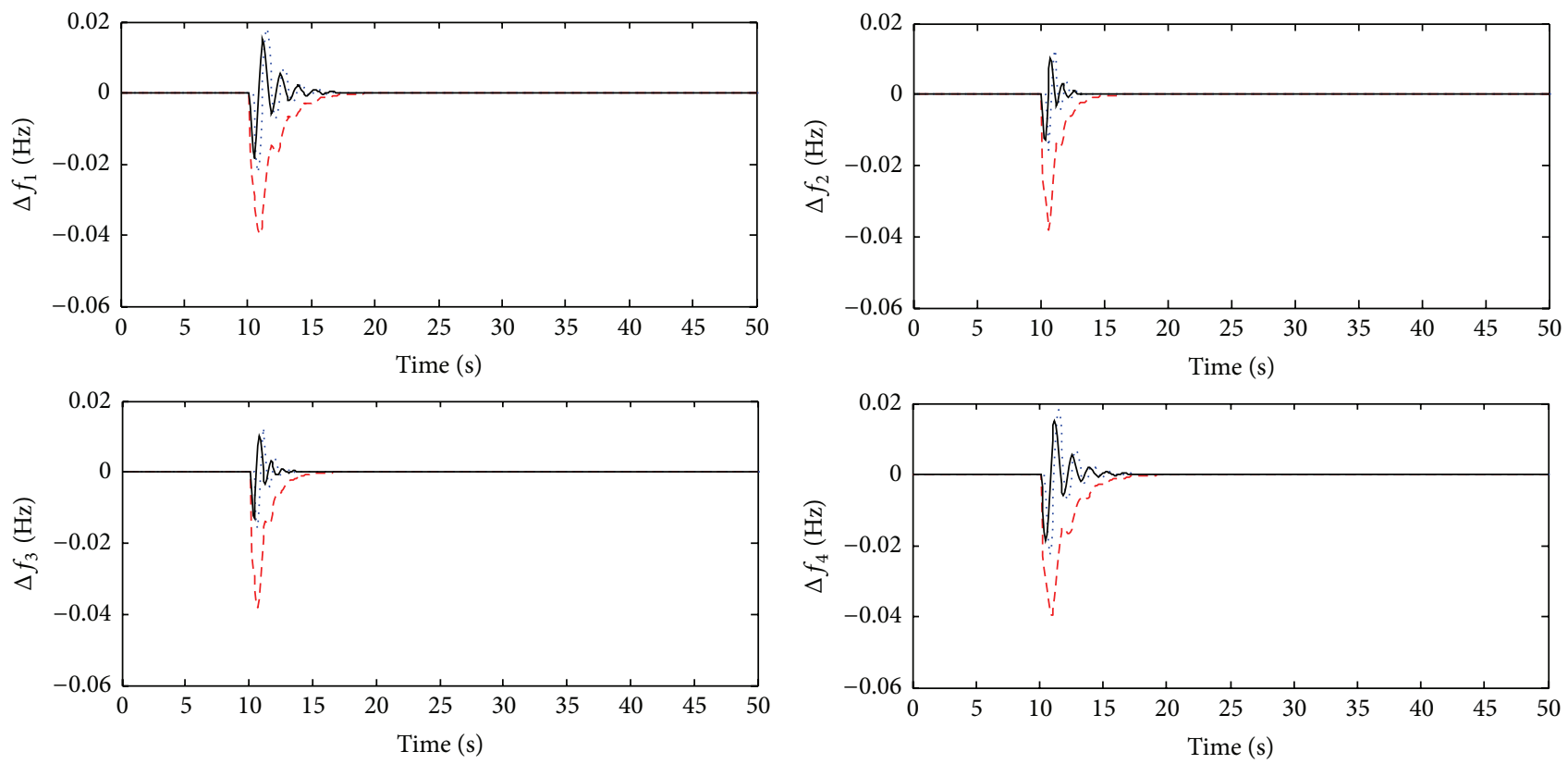

FIGURE 10: Response of frequency deviation to step load disturbance in Case 1: distributed MPC (solid line), centralized MPC (dotted line), and decentralized MPC (dashed line).

TABLE 2: Cost of the different strategies.

\begin{tabular}{lc}
\hline Strategy & Cost [20] \\
\hline Centralized MPC & 0.10 \\
Decentralized MPC & 0.083 \\
Distributed MPC & 0.078 \\
\hline
\end{tabular}

Figure 1 is subjected to step load disturbances as give in (41) at $t=10 \mathrm{~s}$. Consider

$$
\Delta P_{d 1}=\Delta P_{d 2}=\Delta P_{d 3}=\Delta P_{d 4}=0.1
$$

Figure 10 shows the simulation results of distributed MPC, centralized MPC, and decentralized MPC without wind turbine participation and only conventional integrator systems. The relative performance of distributed MPC, centralized MPC, and decentralized MPC rejecting the load disturbance in each area in Figure 10 is denoted by solid, dotted, and dashed lines, respectively. It has been noticed that the closed-loop trajectory of distributed MPC obtained by algorithm is little fast and almost indistinguishable from the closed-loop trajectory of centralized MPC. It successfully improves the dynamic response of area frequencies compared with decentralized MPC.

The control costs defined by [20] for different strategies are listed in Table 2. It is obviously seen that the DMPC controller needs nearly as much CPU time as decentralized MPC controller and significantly less CPU time than centralized MPC controllers. The proposed DMPC algorithm has significant computational advantages when compared to centralized MPC while achieving the best performance.
Case 2 (response to step load change with wind turbines participation). Wind turbine is present and it will provide active power support in the event of grid frequency deviation. An event is simulated in which a system shown in Figure 1 is subjected to step load disturbances as give in (41) at $t=10 \mathrm{~s}$. Mean wind speed is assumed to be $17 \mathrm{~m} / \mathrm{s}$ in area 1 .

In Figures 11 and 12, the behavior for the frequency is presented for Case 2 where the wind turbines are participating in load frequency control. The results from top to the bottom in Figure 11 are the frequency deviations for area 1 to area 4 and in Figure 12 are six tie-lines power change. In simulation, it is obvious that both the DMPC and the centralized MPC converge rapidly and drive the local frequency changes and tie-line power deviation to zero. The wind turbines that have participated in the interconnected power system do not affect the performance of the power system under distributed MPC and centralized MPC while satisfying all the physical constraints, for example, the GRC, the limit of the governors, and load step change constraints. However, with decentralized MPC, the rapid convergence cannot be guaranteed in the presence of wind turbines in area 1. This confirms the performance advantage of the proposed distributed model predictive control algorithm.

Figure 13 shows the dynamic response of active power deviation $\Delta P_{e}$ and rotor speed $\omega_{g}$ of wind turbine while participating in the load frequency control. When the control is activated, the frequency deviation becomes zero which consequently eliminated the additional active power deviation $\Delta P_{e}$ and wind turbine is driven to operate again at the optimal rotor speed $\omega_{g}$. It may be noted here that an increase in power step on top of the converter further reduces the rotor speed, thereby transferring more kinetic power to reduce the frequency dip. As shown in this figure, the distributed MPC 

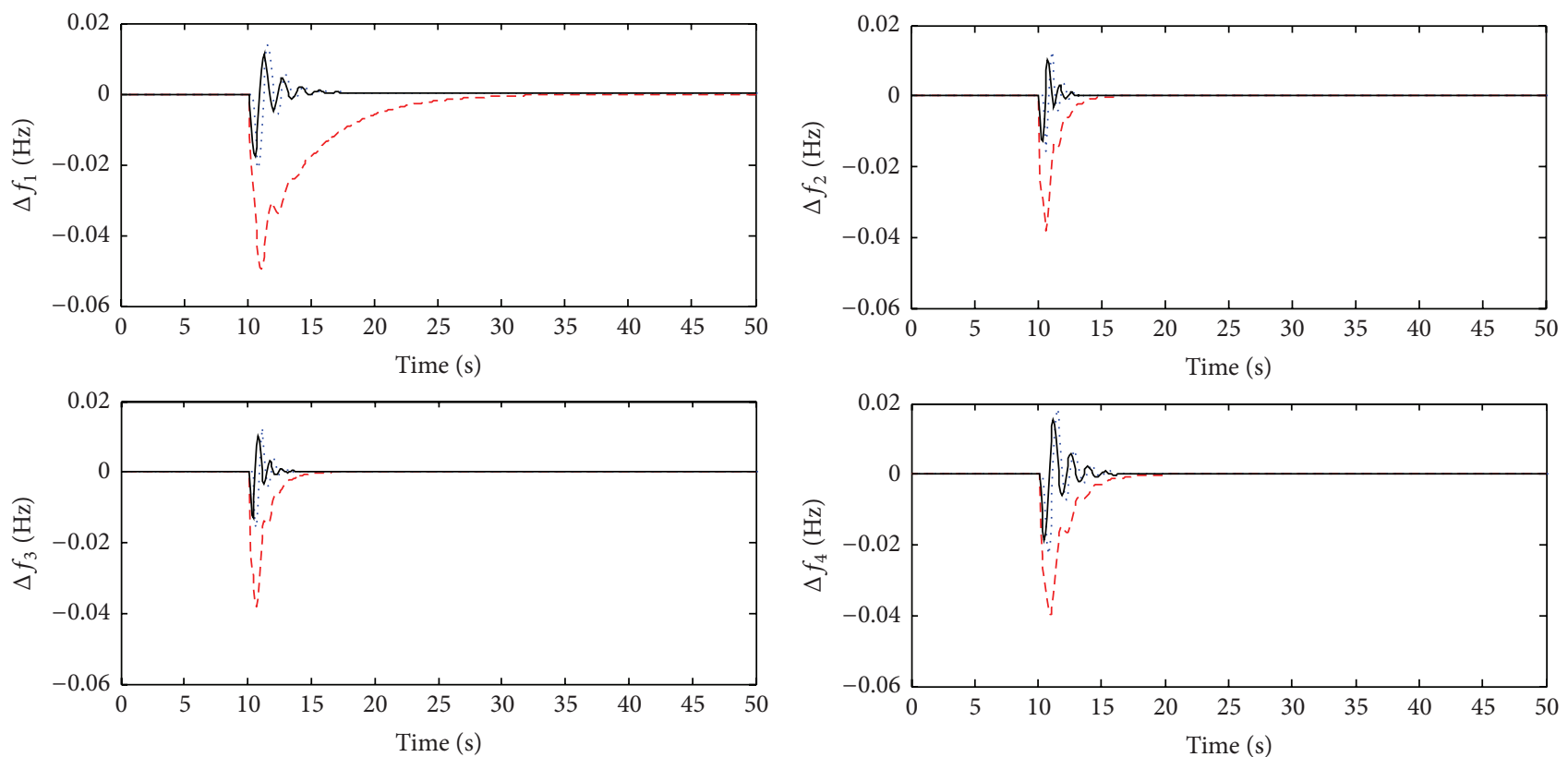

Figure 11: Response of frequency deviation to step load disturbance in Case 2: distributed MPC (solid line), centralized MPC (dotted line), and decentralized MPC (dashed line).
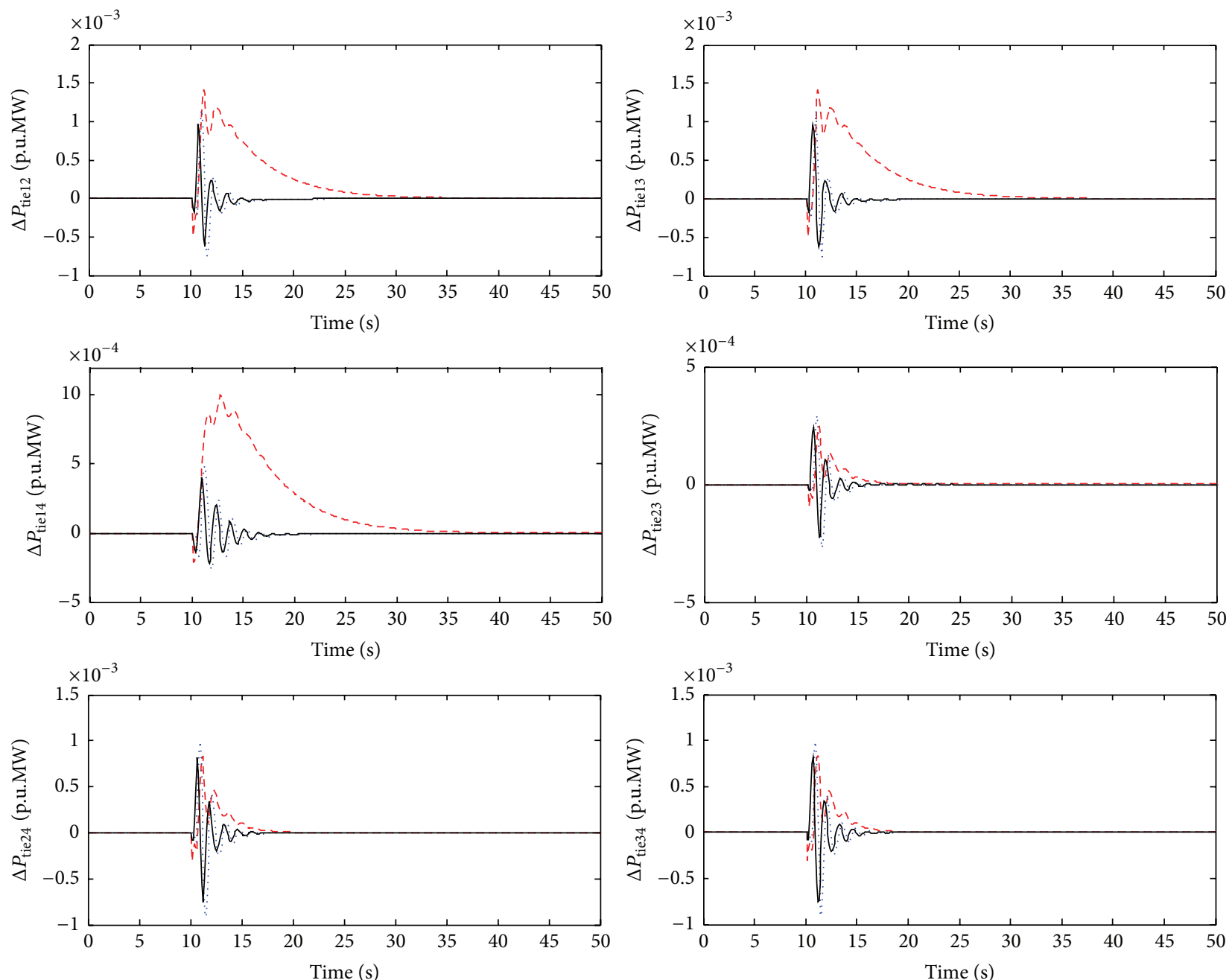

FIGURE 12: Response of tie-line active power deviation in Case 2: distributed MPC (solid line), centralized MPC (dotted line), and decentralized MPC (dashed line). 

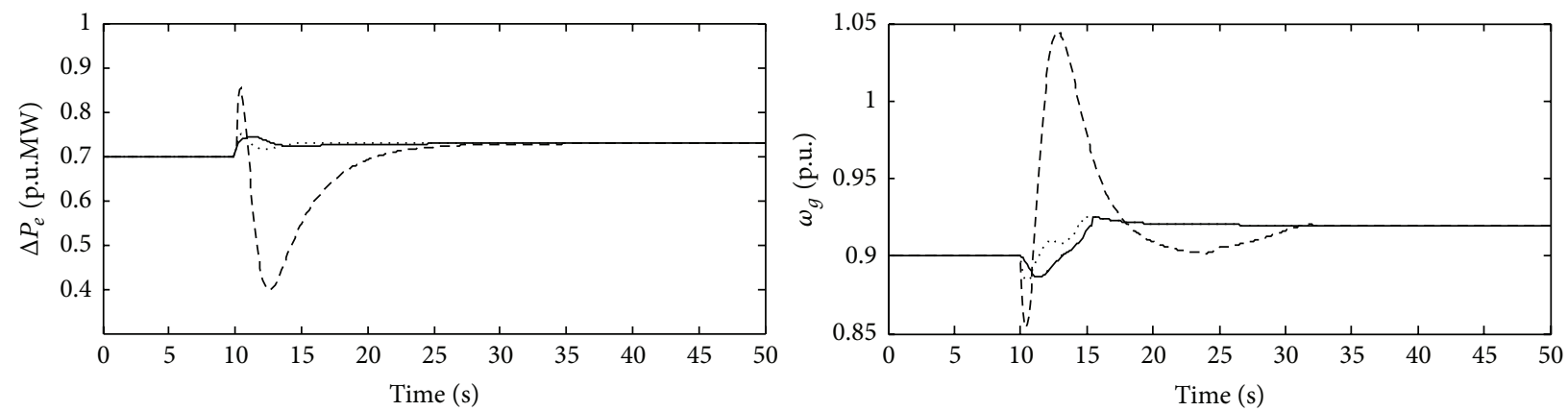

FIGURE 13: Wind turbine response of electrical power and rotor speed in Case 2: distributed MPC (solid line), centralized MPC (dotted line), and decentralized MPC (dashed line).
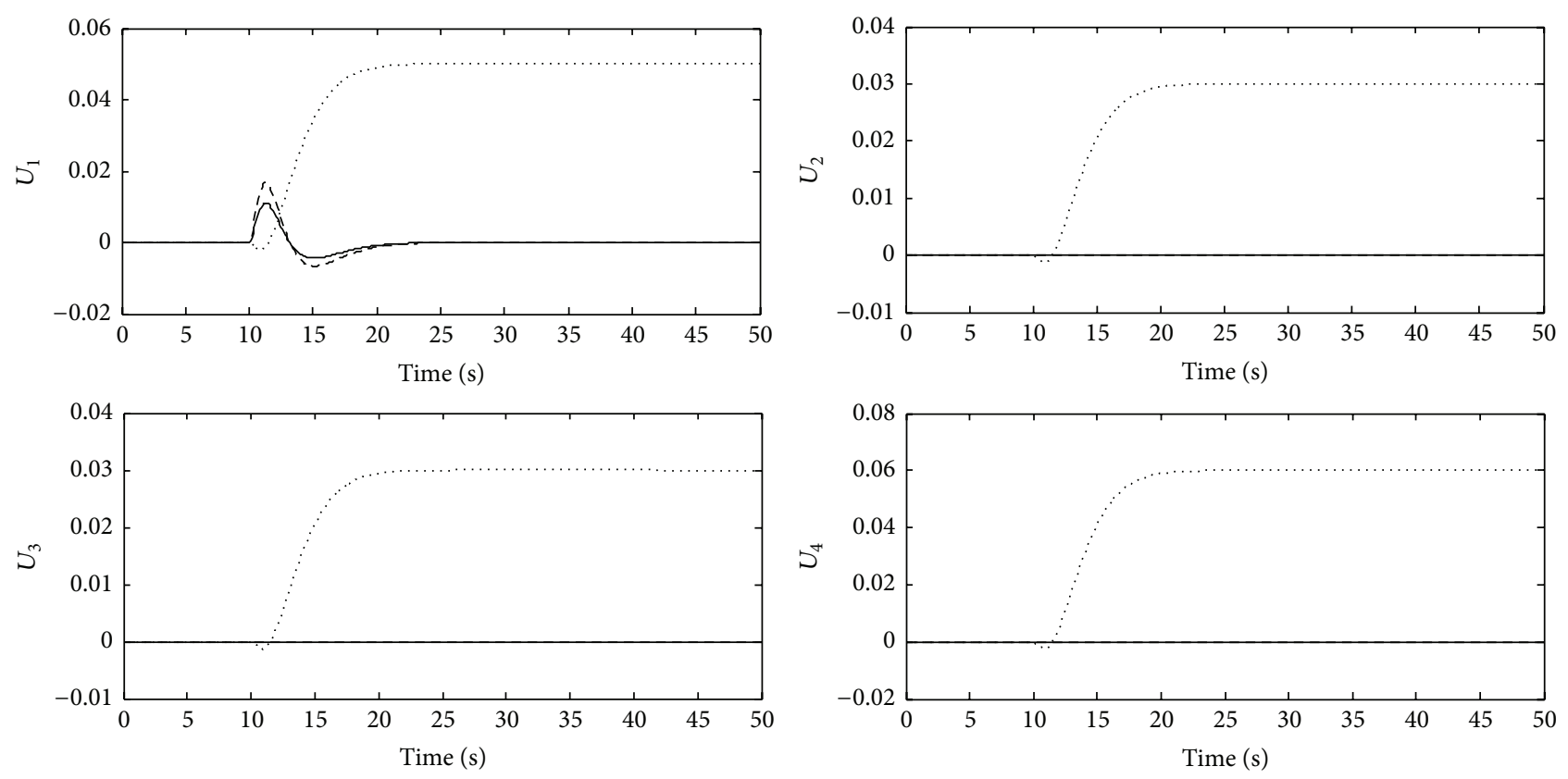

FIGURE 14: Control signal of distributed MPC in Case 2: $\Delta \theta_{\text {ref }}$ in area 1 (solid line), $\Delta P_{c i}$ in four areas (dotted line), and $\Delta T_{g}$ in area 1 (dashed line).

in the presence of wind turbine has desirable performance in comparison to centralized MPC and decentralized MPC.

The distributed MPC control actions as shown in Figure 14, $\Delta \theta_{\text {ref }}, \Delta P_{c i}$, and $\Delta T_{g}$ in four areas are depicted as solid, dotted, and dashed line, respectively. $\Delta \theta_{\text {ref }}$ and $\Delta T_{g}$ are the control signals of wind turbine in area 1 , and $\Delta P_{c i}$ is the control signal of traditional power plants in the four areas. Figure 15 shows the generating outputs of traditional plants.

\section{Conclusions}

In this paper, a DMPC scheme is presented for the LFC of a four-area interconnected power system with wind turbines. The state and input constraints including the valve position limit on the governor and the GRCs were incorporated into the system design. In our scheme, each control area has a local MPC controller, in which the four controllers coordinated with each other by exchanging their information. Comparisons of response to step load change and computational burden have been made between DMPC, centralized MPC, and decentralized MPC. The simulation results verified the reliability of the DMPC for achieving a performance that has advantages over the centralized MPC and distributed MPC in the presence of load changes. Moreover, the proposed DMPC scheme can guarantee a good performance under the wind turbines participation in LFC. Future work will be the extension of the proposed DMPC to different renewable energy contained LFC, since the greater utilization of intermittent renewable resources will induce greater power flow fluctuations.

\section{Conflict of Interests}

The authors declare that there is no conflict of interests regarding the publication of this paper. 

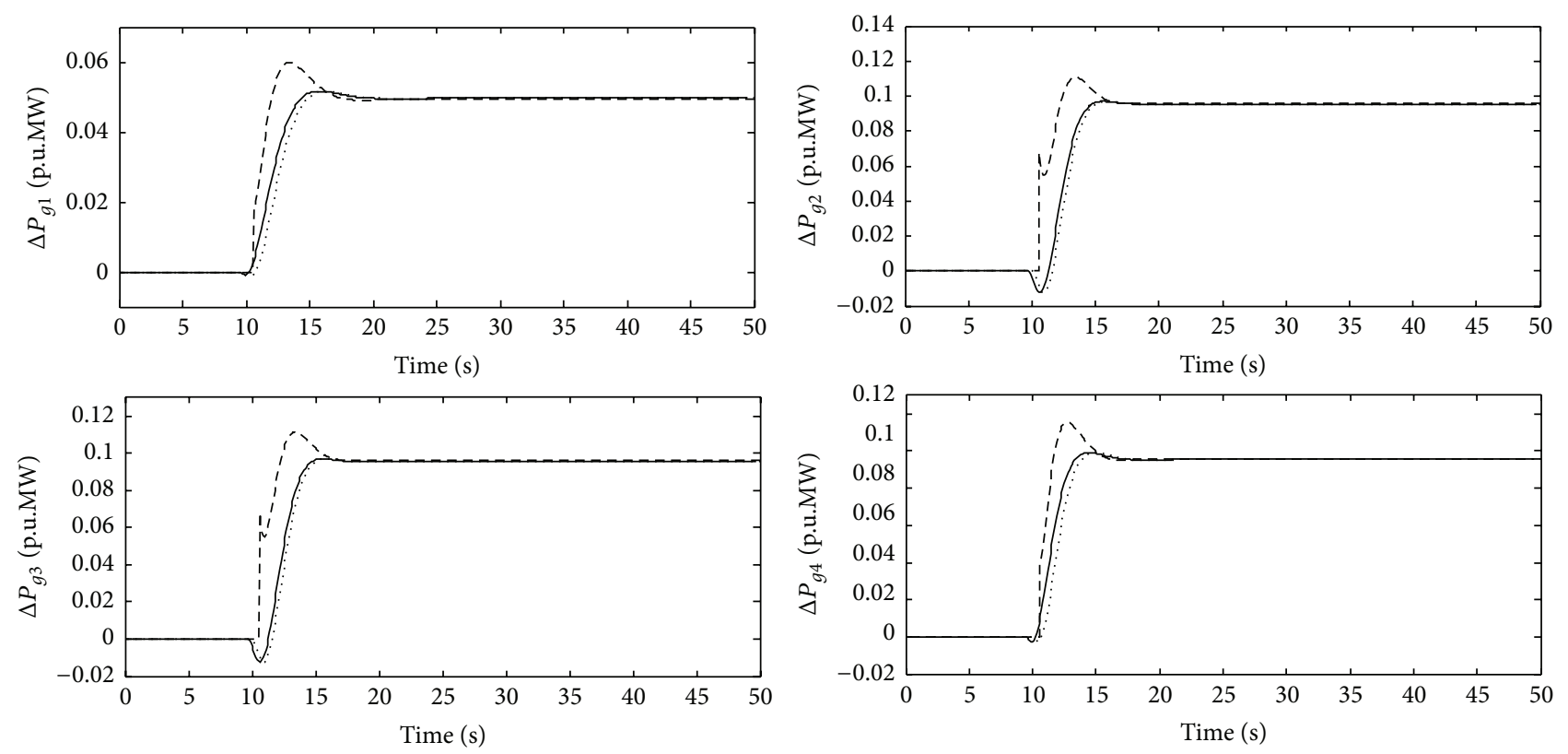

FIGURE 15: Response of generated power deviation in Case 2: distributed MPC (solid line), centralized MPC (dotted line), and decentralized MPC (dashed line).

\section{Acknowledgments}

This project was supported by National Natural Science Foundation of China under Grants 60974051 and 61273144, Natural Science Foundation of Beijing under Grant 4122071, Scientific Technology Research and Development Plan Project of Tangshan under Grant 13130298b, and Scientific Technology Research and Development Plan Project of Hebei under Grant z2014070.

\section{References}

[1] Global Wind Energy Council, Global Wind Report on Annual Market, Global Wind Energy Council, 2014.

[2] H. Bevrani, F. Daneshfar, and R. P. Daneshmand, "Intelligent power system frequency regulations concerning the integration of wind power units," in Wind Power Systems: Applications of Computational Intelligence, L. F. Wang, C. Singh, and A. Kusiak, Eds., Green Energy and Technology, pp. 407-437, Springer, Berlin, Germany, 2010.

[3] X. Yingcheng and T. Nengling, "Review of contribution to frequency control through variable speed wind turbine," Renewable Energy, vol. 36, no. 6, pp. 1671-1677, 2011.

[4] Y.-Z. Sun, Z.-S. Zhang, G.-J. Li, and J. Lin, "Review on frequency control of power systems with wind power penetration," in Proceedings of the International Conference on Power System Technology, pp. 1-8, IEEE, Hangzhou, China, October 2010.

[5] S. K. Pandey, S. R. Mohanty, and N. Kishor, "A literature survey on load-frequency control for conventional and distribution generation power systems," Renewable and Sustainable Energy Reviews, vol. 25, pp. 318-334, 2013.

[6] F. Díaz-González, M. Hau, A. Sumper, and O. Gomis-Bellmunt, "Participation of wind power plants in system frequency control: review of grid code requirements and control methods,"
Renewable and Sustainable Energy Reviews, vol. 34, pp. 551-564, 2014.

[7] H. Shayeghi, H. A. Shayanfar, and A. Jalili, "Load frequency control strategies: a state-of-the-art survey for the researcher," Energy Conversion and Management, vol. 50, no. 2, pp. 344-353, 2009.

[8] L.-R. Chang-Chien, C.-C. Sun, and Y.-J. Yeh, "Modeling of wind farm participation in AGC," IEEE Transactions on Power Systems, vol. 29, no. 3, pp. 1204-1211, 2014.

[9] H. Bevrani and P. R. Daneshmand, "Fuzzy logic-based loadfrequency control concerning high penetration of wind turbines," IEEE Systems Journal, vol. 6, no. 1, pp. 173-180, 2012.

[10] M. H. Variani and K. Tomsovic, "Distributed automatic generation control using flatness-based approach for high penetration of wind generation," IEEE Transactions on Power Systems, vol. 28, no. 3, pp. 3002-3009, 2013.

[11] X. J. Liu, P. Guan, and C. W. Chan, "Nonlinear multivariable power plant coordinate control by constrained predictive scheme," IEEE Transactions on Control Systems Technology, vol. 18, no. 5, pp. 1116-1125, 2010.

[12] X.-J. Liu and C. W. Chan, "Neuro-fuzzy generalized predictive control of boiler steam temperature," IEEE Transactions on Energy Conversion, vol. 21, no. 4, pp. 900-908, 2006.

[13] X. J. Liu and X. B. Kong, "Nonlinear fuzzy model predictive iterative learning control for drum-type boiler-turbine system," Journal of Process Control, vol. 23, no. 8, pp. 1023-1040, 2013.

[14] D. Rerkpreedapong, N. Atic, and A. Feliachi, "Economy oriented model predictive load frequency control," in Proceedings of the Large Engineering Systems Conference on Power Engineering, pp. 12-16, IEEE, Montreal, Canada, May 2003.

[15] X. Liu, X. Kong, and X. Deng, "Power system model predictive load frequency control," in Proceedings of the American Control Conference (ACC '12), pp. 6602-6607, June 2012.

[16] T. H. Mohamed, J. Morel, H. Bevrani, and T. Hiyama, "Model predictive based load frequency control design concerning 
wind turbines," International Journal of Electrical Power \& Energy Systems, vol. 43, no. 1, pp. 859-867, 2012.

[17] T. H. Mohamed, H. Bevrani, A. A. Hassan, and T. Hiyama, "Decentralized model predictive based load frequency control in an interconnected power system," Energy Conversion and Management, vol. 52, no. 2, pp. 1208-1214, 2011.

[18] Y. Zheng, S. Li, and H. Qiu, "Networked coordination-based distributed model predictive control for large-scale system," IEEE Transactions on Control Systems Technology, vol. 21, no. 3, pp. 991-998, 2013.

[19] E. Camponogara and H. F. Scherer, "Distributed optimization for model predictive control of linear dynamic networks with control-input and output constraints," IEEE Transactions on Automation Science and Engineering, vol. 8, no. 1, pp. 233-242, 2011.

[20] A. N. Venkat, I. A. Hiskens, J. B. Rawlings, and S. J. Wright, "Distributed MPC strategies with application to power system automatic generation control," IEEE Transactions on Control Systems Technology, vol. 16, no. 6, pp. 1192-1206, 2008.

[21] M. Mirzaei, N. K. Poulsen, and H. H. Niemann, "Robust model predictive control of a wind turbine," in Proceedings of the American Control Conference (ACC '12), pp. 114-119, Toronto, Canada, June 2012.

[22] M. Yigit, V. C. Gungor, G. Tuna, M. Rangoussi, and E. Fadel, "Power line communication technologies for smart grid applications: a review of advances and challenges," Computer Networks, vol. 70, pp. 366-383, 2014.

[23] M. Ma, H. Chen, X. Liu, and F. Allgöwer, "Moving horizon $H^{\infty}$ control of variable speed wind turbines with actuator saturation," IET Renewable Power Generation, vol. 8, no. 5, article 498, 2014. 

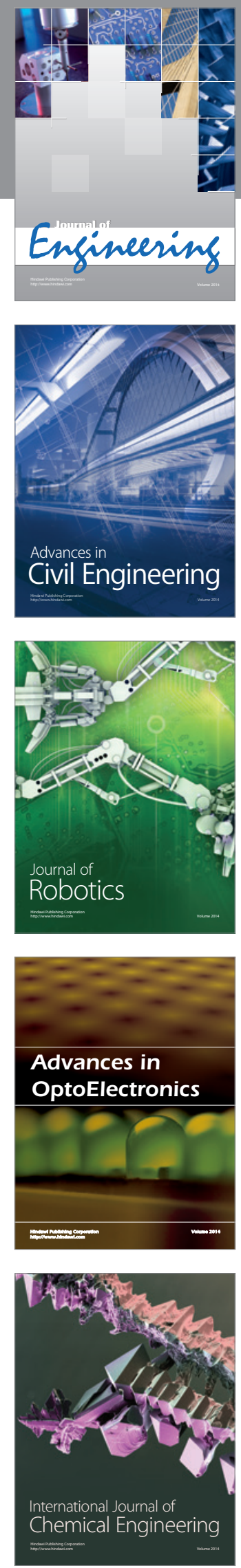

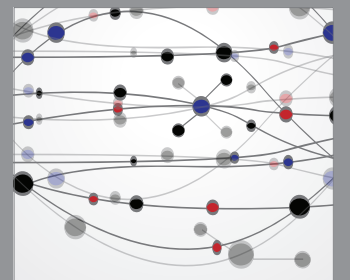

The Scientific World Journal
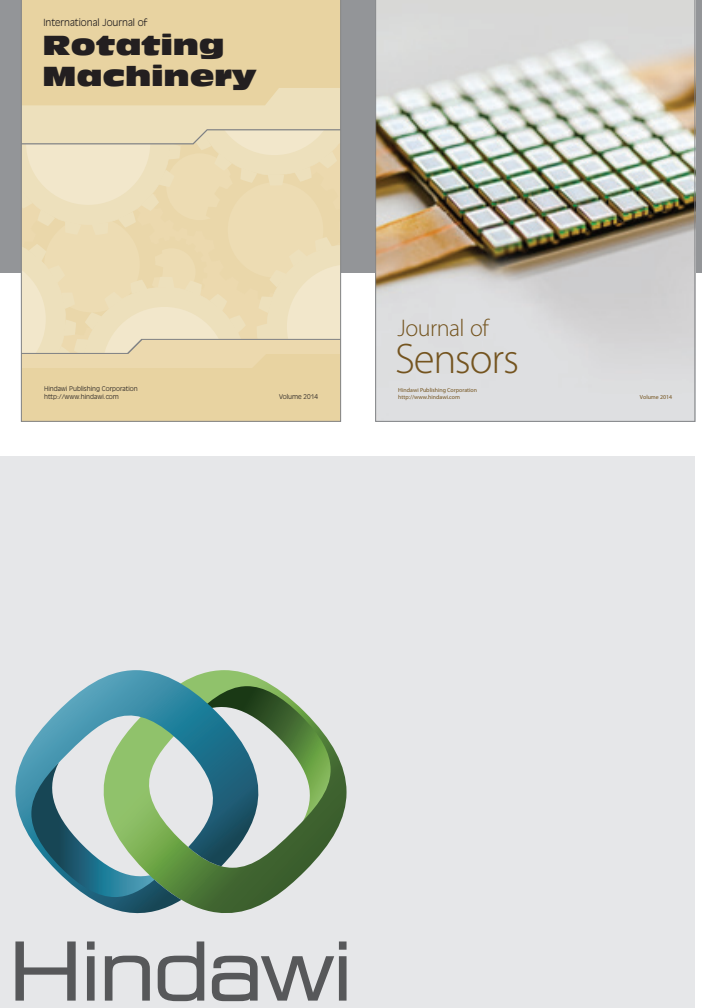

Submit your manuscripts at http://www.hindawi.com
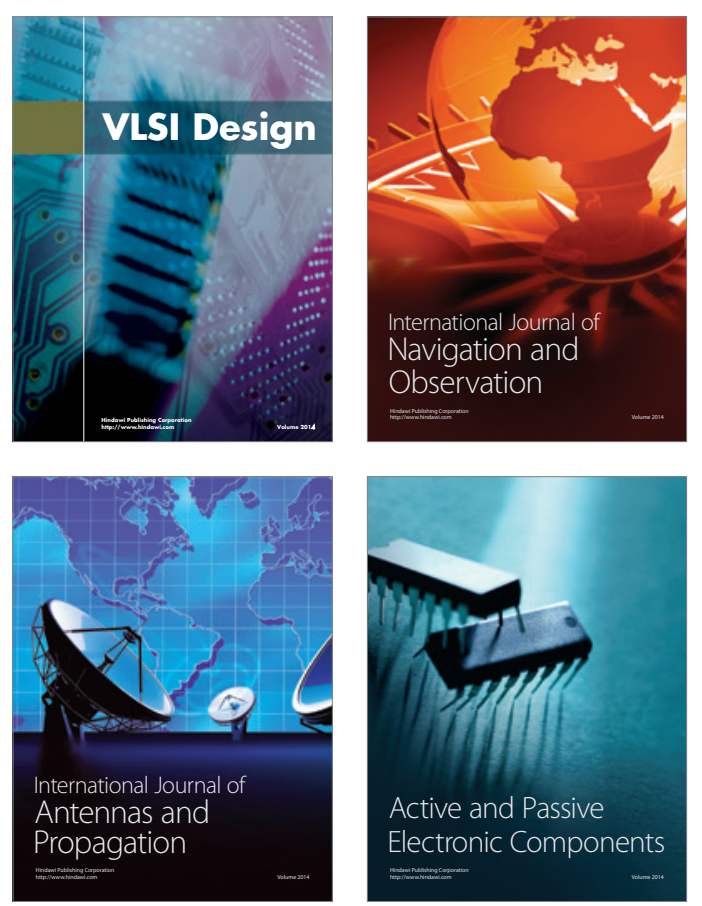
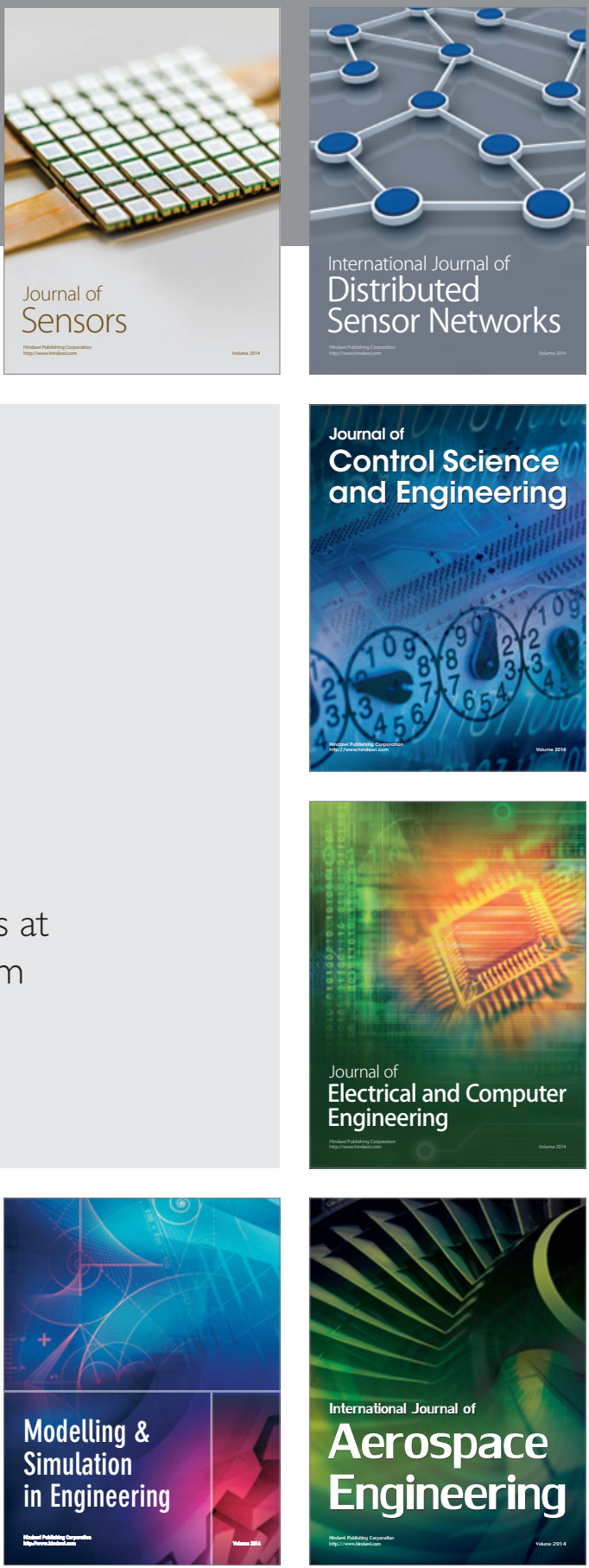

Journal of

Control Science

and Engineering
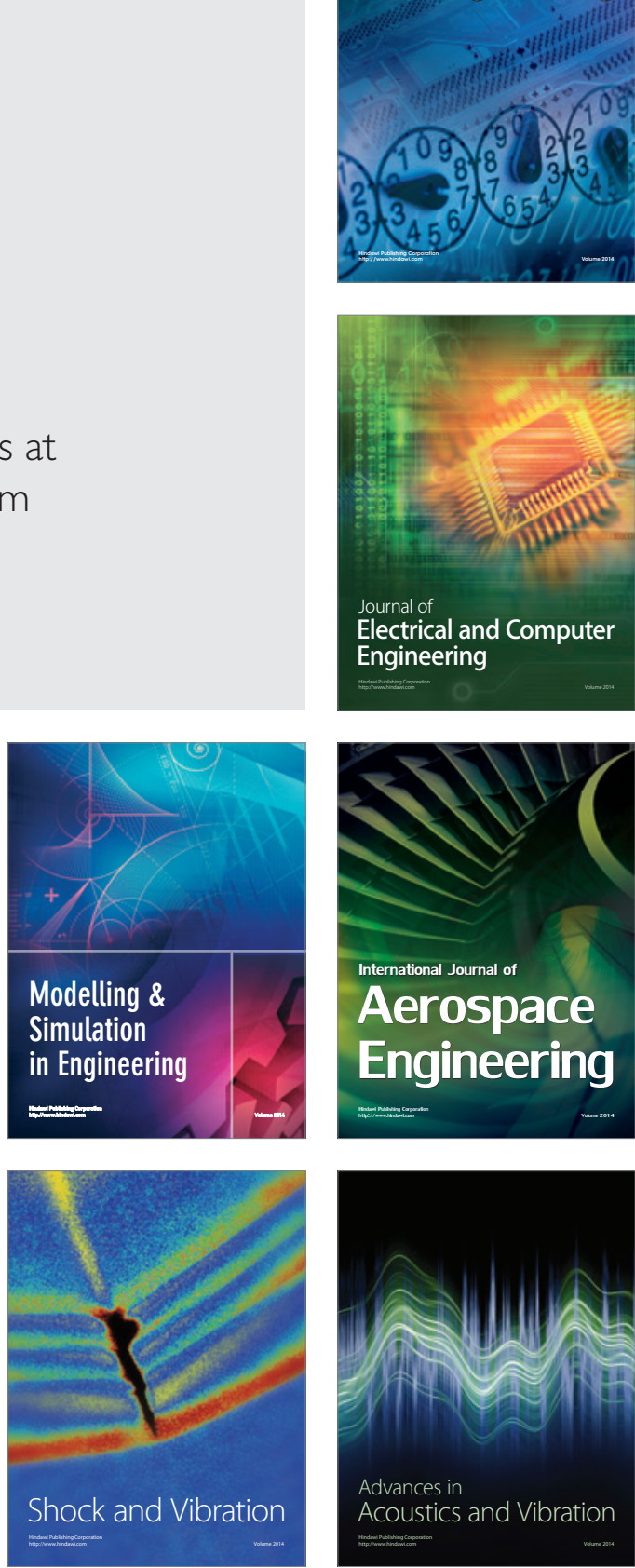